\title{
Contribución al conocimiento de las Chrysomyinae y Toxotarsinae (Diptera: Calliphoridae) de Colombia
}

\author{
Contribution to the knowledge of Chrysomyinae and Toxotarsinae (Diptera: Calliphoridae) \\ from Colombia
}

\author{
Eduardo Amat ${ }^{1,2}$ \\ ${ }^{l}$ Dirección actual: Bolsista de Mestrado CNPq Coordenação de Pesquisas em Entomologia, Instituto Nacional de Pesquisas da Amazônia. Manaus, \\ Amazonas, Brasil. \\ ${ }^{2}$ Instituto de Investigación de Recursos Biológicos Alexander von Humboldt, Claustro de San Agustín. Villa de Leiva, Boyacá, Colombia. \\ *Correspondencia: ecamat@gmail.com; ecamat@inpa.gov.br
}

Resumen. Se presenta información taxonómica, geográfica y ecológica de 21 especies de califóridos de las subfamilias Chrysomyinae y Toxotarsinae encontradas en Colombia. Se proveen claves taxonómicas para la identificación de sus géneros y especies.

Palabras clave: claves, distribución, genitalia, taxonomía, faunística.

\begin{abstract}
Taxonomic, geographical and ecological information of 21 especies of Colombian blowflies of the subfamilies Chrysomyinae and Toxotarsinae is presented. Keys for taxonomical identification of the genera and species are provided.

Key words: Keys, distribution, genitalia, taxonomy, faunistics.
\end{abstract}

\section{Introducción}

Las moscas califóridas (Diptera: Calliphoridae) son principalmente descomponedoras, sarcosaprófagas y/o coprófagas. Su importancia médica y sanitaria radica básicamente en que actúan como agentes de dispersión mecánica de patógenos procedentes de fuentes de infección hacia productos de consumo humano o animal; las larvas de algunas especies pueden causar miasis en mamíferos. Por su grado de asociación con el hombre y su preferencia de hábitat, las especies de califóridas se pueden clasificar como eusinantrópicas (endófilas y exófilas), hemisinantrópicas y asinantrópicas (Povolný, 1971). Por sus hábitos carroñeros, las califóridas han sido utilizados recientemente en el área forense debido a que forman parte de las comunidades de insectos colonizadoras de cadáveres y son el principal grupo de insectos que acuden a la escena de los crímenes. Su ciclo de vida y el ensamblaje de las comunidades aportan datos valiosos para el esclarecimiento de los casos forenses.

La familia Calliphoridae pertenece a la superfamilia Oestroidea (MacAlpine, 1989). Generalmente se considera compuesta de 5 subfamilias (Hall, 1948); sin embargo, existe controversia al respecto, dadas las discrepancias

Recibido: 19 septiembre 2008; aceptado: 06 febrero 2009 acerca del estado taxonómico de algunas subfamilias aún no resueltas. Una discusión detallada acerca de la clasificación de las subfamilias puede ser consultada en Rognes (1986, 1997) y Peris y Gonzales (2005). Las subfamilias presentes en el Neotrópico son Mesembrinellinae, Calliphorinae, Chrysomyinae, Toxotarsinae y Rhiniinae (James, 1970; Peris, 1992; Mello, 2003), esta última ausente en Colombia. La riqueza de especies de la fauna neotropical es considerablemente baja en comparación con otras regiones; solamente se registran 126 especies (Amorim et al., 2002), si se incluye la subfamilia Mesembrinellinae, considerada distintamente por algunos autores como Mesembrinellidae (Guimarães, 1977; Mariluis y Peris, 1984), además de las especies invasoras del género Chrysomya, originarias de África y Asia. En Colombia han sido registradas 30 especies de califóridas (Pape et al., 2004), y se espera la adición de nuevos registros y nuevas especies a la lista, a medida que se extienda su estudio en esta región de América del Sur.

Los califóridos neotropicales han sido objeto de estudio de varios autores. Vale la pena destacar los trabajos de Shannon (1926), quien publicó la sinopsis de los califóridos americanos donde provee de claves taxonómicas preliminares e información biológica para diversas especies. Hall (1948) revisó las especies de califóridos para América del Norte, su obra es referencia 
obligada por ser una revisión completa del material tipo de muchas especies y la reorganización taxonómica de varios grupos. James (1970) publicó el catálogo de la familia Calliphoridae para la región neotropical, en el cual se registraron cerca de 20 especies de Colombia. Mello (1962, 1968) y Guimarães (1977) contribuyeron al conocimiento de la fauna brasileña. Otros trabajos importantes son los de Dear $(1979,1985)$ sobre la subfamilia Toxotarsinae y la tribu Chrysomyini. Mariluis y Peris (1984) publicaron la sinopsis de las especies neotropicales; Carvalho (2000) y Mello (2003), las claves para la identificación de especies en Brasil, y Mariluis (1981), Mariluis y Schnack (2001) y Mariluis y Mulieri (2003) lo hacen para Argentina. Estos últimos incluyen información detallada sobre la distribución geográfica de las especies. Entre los trabajos más recientes, vale la pena mencionar la clave ilustrada de especies para Colombia de Amat et al. (2008) y la clave para la identificación de moscas con interés forense, más comunes en América del Sur, de Carvalho y Mello-Patiu (2008), donde se incluyen varias especies de Calliphoridae. Entre los estudios ecológicos sobre califóridos cabe resaltar el de Greenberg y Szyska (1984) sobre biología, con descripción de estados inmaduros de varias especies neotropicales del Perú. Para este mismo país, Baumgartner y Greenberg (1985) recopilaron importante información ecológica y médica. En la región Amazónica, la ecología de Callihoridae fue estudiada por Paralupi (1992) y Esposito (1999). Adicionalmente, se puede encontrar información de varias especies en un apreciable número de estudios de tipo forense para la region neotropical; entre otros, los compilados por Mavárez-Cardozo et al. (2005).

En la region norte de América del Sur, los califóridos se han estudiado poco. La fauna colombiana no se ha estudiado de manera detallada ni en conjunto; así, muchos registros se restringen a las localidades tipo o a ejemplares recolectados y estudiados por especialistas extranjeros y depositados en sus colecciones.

A pesar de su enorme importancia sanitaria, veterinaria y forense poco se sabe acerca de los estados inmaduros y ecología de las especies colombianas; recientemente Florez y Wolff (2009) describen e ilustran los estados inmaduros de 13 especies colombianas de interés forense. Es necesario resaltar la necesidad de generar y fomentar estudios que abarquen información básica taxonómica, biológica y ecológica para incrementar su conocimiento en el área médica y veterinaria, y consecuentemente un adecuado uso legal y forense. Este estudio pretende contribuir con información básica taxonómica, geográfica, y en algunos casos ecológica, sobre el conocimiento de las especies pertenecientes a las subfamilias Chrysomyinae y Toxotarsinae presentes en el territorio colombiano. Se incluye la morfología de los genitalia del macho, principalmente cercos y surstilos, para la identificación de las especies.

\section{Materiales y métodos}

Se revisaron 604 especímenes provenientes de 21 departamentos de los 33 que conforman Colombia, incluyendo el Distrito Capital, depositados en las colecciones entomológicas del Instituto Alexander von Humboldt, Villa de Leyva, Boyacá (IAvH); Instituto de Ciencias Naturales, Universidad Nacional de Colombia, Bogotá (ICN-MHN); Universidad Nacional de Colombia Sede Medellín, Museo "Francisco Luis Gallego" (MEFLG); Museo de Historia Natural, Pontificia Universidad Javeriana, Bogotá (MPUJ); Colección entomológica del Instituto Nacional de Medicina Legal y Ciencias Forenses, Bogotá (INML-CF); y Museo Entomológico de la Facultad de Agronomía, Universidad Nacional de Colombia, Bogotá (UNAB).

Para el tratamiento taxonómico de los Chrysomyini se sigue lo propuesto por Dear (1985); para las Toxotarsinae, a Lopes y Albuquerque (1982). Las claves taxonómicas se adaptaron o modificaron de Dear (1985), Gonzales-M. D. et al. (1998), Carvalho y Ribeiro (2000), Mariluis y Schnack (2001), Mello (2003) y Amat et al. (2008). Se ilustra el plan corporal (Fig. 1), quetotaxia y venación típica (Figs. 2-3). Algunos caracteres diagnósticos fueron ilustrados, o redibujados de fuentes originales. Se emplea principalmente la terminología de MacAlpine et al. (1981). Los mapas estan elaborados utilizando el software DIVA GIS versión 5.4 (Hijmans et al., 2006), las localidades sin coordenadas geográficas se georrefirieron gracias a los gaceteros geográficos de DIVA GIS (Hijmans et al., 2006) y de la National Geoespatial-Intelligence Agency, disponibles en internet. La figura de distribución altitudinal se elaboró de acuerdo con los registros de menor y mayor altura de cada especie, respectivamente.

\section{Subfamilia Chrysomyinae}

Es de distribución cosmopolita, se encuentra conformada por las tribus Chrysomyini y Phormini, de las cuales sólo la primera se encuentra en el Neotrópico, con 20 especies endémicas y 4 introducidas (Dear 1985). En Colombia fueron registrados 6 géneros. Los Chrysomyiini son sarcosaprófagos y carroñeros, a excepción de las 2 especies parásitas causantes de miasis.

\section{Chrysomya Robienau-Desvoidy}

Hace aproximadamente 3 décadas, este género se introdujo al continente americano; 3 de sus especies $(C$. 
Clave para la identificación de subfamilias de Calliphoridae de Colombia.

1. Subescutelo levemente desarrollado y piloso sobre su superficie ventral (Fig. 4); espiráculo posterior grande con una aleta abierta dorsalmente (Fig. 5); curvatura de la vena M apenas pronunciada (Fig. 6)... ..Mesembrinellinae.

Subescutelo poco o nada desarrollado y sin pilosidad sobre su superficie ventral (Fig. 7); espiráculo posterior normal sin abertura dorsal, variable en tamaño y forma; curvatura de la vena M angulosa (Fig. 8).

2. Base de la vena radial R (vena troncal o vena remigio) dorsalmente desnuda (Fig. 9); ampolla ovoide (Fig. 10). Calliphorinae.

Base de la vena radial R (vena troncal o vena remigio) dorsalmente setosa (Fig. 11); ampolla en forma de riñón (Figs. 12,13)........ .............................................................. 3

3. Base de la vena radial R (vena troncal o vena remigio) ventralmente desnuda...... Chrysomyinae (Chrysomyini).

Base de la vena radial R (vena troncal o vena remigio) ventralmente setosa (Fig. 13) Toxotarsinae.

Clave para la identificación de géneros de Chrysomyinae de Colombia.

1. Ampolla cubierta con pilosidad larga (Fig. 12); calíptero inferior dorsalmente piloso en toda su superficie y subtruncado posteriormente con el borde externo angular (Fig. 14) ...........Chrysomya Robineau-Desvoidy, 1830.

Ampolla cubierta con pilosidad corta (Figs. 10,13); calíptero inferior dorsalmente desnudo o solamente con alguna pilosidad basal y con el borde externo usualmente redondeado (Fig. 15)

2. Parafacial con una fina pilosidad (Fig. 16b) mucho más conspicua en hembras; cerdas dorsocentrales presuturales e intraalares presuturales (Fig. 2) ausentes; mesonoto usualmente con 3 bandas longitudinales (Fig. 17).

Parafacial desnuda; cerdas dorsocentrales presuturales e intraalares (Fig. 2) presentes; mesonoto sin bandas longitudinales.......................................4

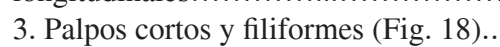
Cochliomyia Townsend, 1915.

Palpos normales y clavados (Fig. 19)............................ Compsomyiops Townsend, 1918.

4. Una cerda acrostical postsutural, cerda supraalar presutural ausente; calíptero inferior desnudo y diverge de la línea borde del escutelo (Fig. 15), margen posterior de la coxa posterior piloso sobre la superficie superior (Fig. 20); ala levemente ahumada en las celdas bc, c, sc, r1, primer tercio de la br y porción anterior de $\mathrm{r} 2+3$; cercos y surstilos del macho en vista posterior como en la figura

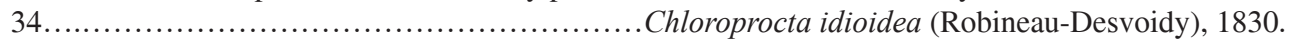

Dos cerdas acrosticales postsuturales; 2 cerdas supraalares presuturales presentes; calíptero inferior desnudo o piloso sobre su mitad basal; margen posterior de la coxa posterior piloso sobre toda su superficie; región costal del ala hialina o con algún patrón alar.

5. Calíptero inferior dorsalmente piloso en su región basal (Fig. 14); 2 cerdas anteriores katepisternales y

1 cerda posterior katespisternal; alas hialinas o levemente oscurecidas en su porción basal.....................................................Paralucilia Brauer y Bergenstamm, 1891.

Calíptero inferior desnudo; 1 cerda anterior katepisternal (algunos especímenes pueden presentar 1 cerda adicional anterior menor que la principal) y 1 cerda posterior katepisternal; alas usualmente con un par de regiones ahumadas; una localizada en la parte proximal cubriendo las celdas c, bc y sc; la otra, cubriendo la porción distal de r1 y r2+3...

Hemilucilia Brauer, 1895.

albiceps [Wiedemann, 1819], C. putoria [Wiedemann, 1818] y C. megacephala [Fabricius, 1794] ) lo hicieron por Brasil y 1 (C. rufifacies [Macquart, 1843] ) por Costa Rica. Más información acerca de esta invasión se encuentra en Guimarães et al. (1978), Guimarães (1979), Jiron (1979), Mariluis (1980, 1983) Baumgartner D.L y Greenberg B (1984), Peris S.V. (1985,1987), entre otros. El primer registro de Chrysomya para Colombia fue el de C. putoria (Wiedemann, 1818) encontrada en Leticia (Amazonas), documentado por Baumgartner y Greenberg (1984). Más adelante, Baumgartner (1988) registró C. albiceps (Wiedemann, 1819) y C. megacephala (Fabricius, 1794) en Venezuela y sugirió como establecimiento de estas especies el lado oriental de los Andes tropicales, presumiblemente del colombiano. Wolff et al. (2001) registran C. albiceps (Wiedemann, 1819) en Medellín (Antioquia); Barreto et al. (2002), C. rufifacies Macquart, 1843), en Cali (Valle del Cauca), y Pape et al. (2004) registran C. megacephala 
(Fabricius, 1794) en Antioquia, Bolívar, Sucre y Valle del Cauca, así como C. albiceps (Wiedemann, 1819) en Antioquia, Boyacá, Cundinamarca, Santander y Sucre.

Ha existido confusión con el estado taxonómico de $C$. putoria (Wiedemann, 1818) y C. chloropyga (Wiedemann, 1818), consideradas por algunos autores como formas diferentes de una misma especie; sin embargo, Rognes y Paterson (2005) reconocen 2 especies claramente definidas después de un detallado estudio sobre su morfología y distribución. De la misma manera sucede con C. albiceps (Wiedemann, 1819) y C. rufifacies (Macquart, 1843), su estado taxonómico es aún objeto de discusión. Según Dear (1985), estas 2 especies difieren en la presencia de la cerda proestigmática; sin embargo, Zumpt (1965) las separa por la presencia de una larga cerda proestigmática, refiriéndose a la cerda proepimeral, según la terminología de MacAlpine (1981). Los especímenes revisados en este estudio siguen la descripción de este último autor.

En Colombia C. megacephala (Fabricius, 1794) se encontró principalmente en la zona andina y en el Caribe entre los 0 y $2600 \mathrm{~m}$ de altitud, mientras $C$. albiceps (Wiedemann, 1819) en la zona andina y en la Orinoquia, distribuidas principalmente entre los 340 y $2800 \mathrm{~m}$ de altitud (Figs. 44-50). Chrysomya putoria (Wiedemann, 1819) parece distribuirse principalmente en las selvas amazónicas y bosques de galería en la Orinoquia, tiende al comportamiento asinantrópico y Esposito (1999) la menciona como poco común para la Amazonia brasileña. Ningún ejemplar de C. rufifacies (Macquart, 1843) se revisó en este estudio; sin embargo, está incluida en la clave debido al previo registro de Barreto et al. (2002), lo que hace necesario confirmar la presencia de estas 4 especies introducidas, un hecho particular, porque no se tiene antecedente de su presencia en un mismo país. Colombia pudo haberse convertido en el punto de convergencia en el continente americano; por tanto, es imperativo promover estudios ecológicos si se tiene en cuenta que $C$. rufifacies
(Macquart, 1843) y C. putoria (Wiedemann, 1819) tienen nichos ecológicos similares y hasta el momento no se han encontrado en simpatría (Peris 1987).

Material revisado. Chrysomya albiceps (Wiedemann, 1819): COLOMBIA. Bogotá D.C., 31.x.2004. L. Latorre y S. Jiménez leg. (3 † INML-CF); Idem. 25.vii.2003. G. Camacho leg. ( 9 INML-CF); Idem, excepto: 02.ix.2002 (1 † INML-CF); Usaquén 26.vii.2004 A. Segura Leg.

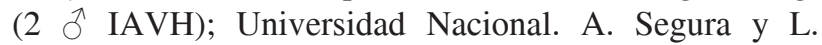
Cañón leg. (1 O INML-CF); Parque Nacional, Estación de Carabineros. 07.i.2003. L. Latorre y S. Jiménez leg. (2 \& INML-CF); Idem, excepto: 17.i.2003 (2 q INMLCF); Idem, excepto: 09.ii.2003 (2 + INML-CF); Idem, excepto: 10.xii.2002. (1 9 INML-CF); Idem, excepto: 11.xiii.2002 (1 † INML-CF); Idem, excepto: 28.i.2003 (1 $\precsim$ INML-CF); Idem, excepto: 07.i.2003 (1 $ぇ$ INMLCF); Idem, excepto: 27.i.2003 (1 ठ INML-CF); Idem, excepto: 11.xii.2002 (1 ठ INML-CF); Idem, excepto: 29.xii.2002 (1 §̂ INML-CF); Idem, excepto: 18.i.2003 (1 $\lesssim$ INML-CF); Instituto Nacional de Medicina Legal y Ciencias Forenses. 20.ix.2002. G. Camacho leg. (1 $q$ INML-CF); Escuela General Santander. 15.ii.2005. K. Zamudio leg. (1 ð INML-CF); Idem, excepto: 03.ii.2001

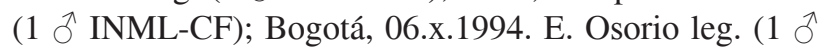
UNAB). Boyacá, Tunja. 21.ii.1994. O. Rivera leg. (1 q UNAB); Villa de Leiva. 15.ii.1989. E. Vargas leg. (1 $\hat{\sigma}$ UNAB). Caldas, Cañaveral La Mula 345m i.2002 rastrojo E. Martínez (1 §̧ MEFLG). Cundinamarca, Mun. La Mesa vereda el Espino 4'38'09' 'N 74²7'59''O $1300 \mathrm{~m}$ en Carroña 0031 18.xii.2002 JJW Leg, (2 ๆ AVH); Cáqueza, Vereda Ubatoque. 1746m. 06.viii.1989. L. Moreno leg. (1 † ICN MHN) ; Mosquera, Corpoica-Tibaitatá. 4²4’ N 74²12'O. 2543m. 25.vi.2001. G. Camacho y F. Ospina leg. (1 † ICN MHN) ; Idem. 26.vi.2001. G. Camacho y F. Ospina leg. (1 9 ICN MHN) ; 1 q , Idem. 27.vi.2001. G. Camacho y F. Ospina leg. (1 9 ICN MHN) ; Idem 30.vi.2001. G. Camacho y F. Ospina leg. ( 3 ICN MHN);

Clave para la identificación de especies de Chrysomya de Colombia.

1. Espiráculo anterior (Fig. 1) negro, o castaño oscuro; macho con las facetas agrandadas notablemente; cercos y surstilos en vista

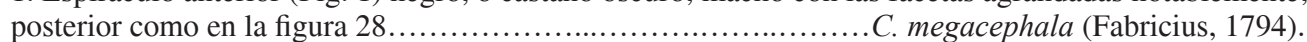

Espiráculo anterior (Fig. 1) blanco o de coloración cremosa; macho con las facetas de tamaño normal...................................................... 2

2. Macho con la porción más estrecha de la frente, menor que el diámetro del ocelo anterior (Fig. 21); cercos y surstilos en vista posterior como en la figura 29; hembra con 2 cerdas frontoorbitales proyectadas lateralmente (Fig. 16a); quinto tergo sin incisión dorsal (Fig. 22)....................................................................... putoria (Wiedemann, 1818).

Macho con la porción más estrecha de la frente, 2 veces el diámetro del ocelo anterior (Fig. 23); hembra sin cerdas frontoorbitales; quinto tergo con incisión dorsal (Fig. 24).......................... 3

3. Una cerda proepimeral (o estigmática) presente (Fig. 25a), 2-4 cerdas proepisternales presentes (Fig. 25b) ...C. rufifacies (Macquart, 1843).

Cerda proepimeral (o estigmática) ausente (Fig. 26a), $4-6$ cerdas proepisternales presentes (Fig. 26b), cercos y surstilos del macho en vista posterior como en la figura $27 \ldots$ C. albiceps (Wiedemann, 1819). 
Idem, 30.vi.2001. G. Camacho y F. Ospina leg. (1 ð̊ ICN MHN); Soacha. 15.vi.1979. H. Jiménez leg. (1 9 UNAB); Caqueza. 17.v.1997. X. Medina leg. (1 $q$ UNAB); La Calera. 23.viii.1992. J. Zuleta leg. (1 9 UNAB); La Mesa. 13.xi.1993. F. Barrera leg. (1 $\lesssim$ UNAB) Tocaima. 23.ix.1994. C. Ferro leg. (1 §̋ UNAB); La Mesa. 20.x.1996. C. Pinzón leg. (1 đ UNAB). Meta, San Martín, Finca El Caduceo 341'40', N 7341'37' 'O 450 m Trampa Pescado 18-xii.2005 E. Amat leg. (3 § IAVH); Idem ,excepto: 18-xii.2005 (3 ○ IAVH); Idem, excepto: 19-xii.2005 (5 q IAVH); San Martín. 17.viii.1997. O. Fuentes leg. (1 UNAB). Santander, Bucaramanga, 11.iv.1995. G. Ardila leg. (1 $q$ UNAB). Vichada, Municipio Cumaribo Cgto. Santa Rita PNN El tuparro 5'21'20'’N 6801'28' O 135 Bosque de cerro Trampa de interceptación de vuelo \# T2 18-21.ii.2004 I. Quintero y E. González leg. (1 §ิ IAVH); Idem, 5'20'48' 'N 6751'36' O 135m Bosque de cerro Trampa Exc humano \# T2 18-21.ii.2004 I. Quintero y E. González Leg, (1 9 IAVH).

Chrysomya megacephala (Fabricius, 1794): COLOMBIA. Antioquia, Copacabana relleno sanitario curva de rodas pastizal Van someren Ridon $1648 \mathrm{~m}$ Trampas pegantes para moscas 16.x.2002 J. Pulgarin leg. (4 क 8 ઈ MEFLG); Idem, excepto: 30.x.2002 Feromonas (1 92 2 MEFLG);Idem, excepto: malaise 24.x.2002 D. Acevedo (1 q MEFLG). Bolívar, Archipiélago de San Bernardo Isla Múcura 946’N 7552'O $10 \mathrm{~m}$ Trampa pescado 05.i.2004 E. Amat y C. Flores leg. (18 § IAVH) ; Idem, excepto: 05.i.2004 (62 + IAVH). Boyacá, Villa de Leiva Vereda Centro 5'38'19' N 73³1'42' O 2400 m Manual 20-24.vi.2005 Z. Reyes leg. (2 §̂ IAVH); Idem, excepto: 27.vi.2005 (2 ᄋ IAVH); Idem, excepto: 20-24.vi.2005 (2 q IAVH); Idem, excepto: Trampa pescado 2.i.2006 E. Amat leg. (4 $\rightarrow$ IAVH). Caldas, Cañaveral la mula $380 \mathrm{~m}$ Bosque secundario i.2002 E. Martínez leg. (1 q MEFLG). Guajira, Cerrejón Campamento Mushaisa 240 m v.2006 M. Reyes leg. (3 92 ô IAVH). Chrysomya putoria (Wiedemann, 1818): COLOMBIA. Vichada, Cumaribo, Selva de Mataven, Comunidad de Sarrapia, 03.iv.2007, $04^{\circ} 29^{\prime} 25^{\prime}$ 'N 67'52'19'O, 240 m, F.Forero leg. Trampa de pescado (10 $+3 \curvearrowright$ IAVH).

\section{Cochliomyia Townsend}

Género endémico de la región neotropical, con 4 especies (Dear 1985). En Colombia solamente se registran $C$. macellaria (Fabricius, 1775) y C. hominivorax (Coquerel, 1858), ampliamente distribuidas en la región neotropical. El género es muy interesante por su importancia médica, veterinaria y económica. Cochliomyia hominivorax (Coquerel, 1858); ha sido objeto de decenas de estudios por ser la mosca causante de la principal miasis en el Neotrópico. Sus larvas son parásitos obligados de tejidos vivos (biontófagas) de mamíferos, incluido el hombre; se considera un problema serio para animales domésticos, ganado vacuno, ovino y caprino (Guimaraes et al., 1983). Posiblemente ésta sea la causa de su escasez en colecciones, pues le resultan de poco interés los cebos utilizados convencionalmente para colecta de moscas descomponedoras.

Cochliomyia macellaria no tiene importancia económica, sus larvas son principalmente saprófagas carroñeras y pueden llegar a ser invasoras secundarias de heridas (necrobiontófagas) (Guimarães et al., 1983). Recientemente Trigo (2006) ofrece nuevos caracteres diagnósticos para la identificación de los estados inmaduros incluyendo su pupario. Se distribuye ampliamente en la franja neotropical y puede llegar a ser una especie muy común, sobre todo en la región Amazónica (Esposito, 1999). Algunos autores sugieren la disminución de sus poblaciones debido a la intensa competencia y similitud de nichos con las especies invasoras del género Chrysomya (Baumgartner y Greenberg 1984, Peris 1987); sin embargo, este hecho aún es tópico de interés y estudio.

Cochliomyia hominivorax es una especie rara en colecciones biológicas. En este estudio, no se encontró ni se revisó ningún ejemplar; sin embargo, se presume que está distribuida ampliamente en Colombia. Cochliomyia macellaria se encontró en todas las regiones naturales de Colombia hasta los $2500 \mathrm{~m}$ de altura (Figs. 45-50).

Clave para la identificación de especies de Cochliomyia en Colombia.

1. Quinto tergo con un par de manchas pruinosas plateadas laterales; hembra con un par de cerdas frontorbitales (Fig. 16a) presentes, pequeñas cerdas de las placas frontorbitales (Fig. 16c) blancas o amarillas, basicosta (Fig. 3) de la hembra amarilla, cercos y surstilos del macho en vista posterior como en la figura $31 . .$. C. macellaria (Fabricius, 1775).

Quinto tergo sin manchas pruinosas laterales, hembra sin cerdas frontoorbitales, pequeñas cerdas de las placas frontales (Fig. 16c) negras, basicosta (Fig. 3) de la hembra castaño oscuro, cercos y surstilos del macho en vista posterior como en la figura

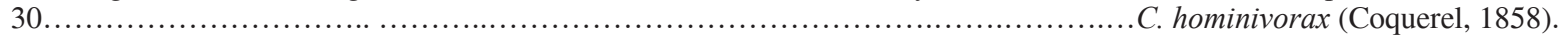


Material revisado. Cochliomyia macellaria (Fabricius, 1775): COLOMBIA. Amazonas, río Igara Paraná, 15 km de la Chorrera. 16.iv.1975. J. Idrobo y C. Sáenz leg. (10 o ICN-MHN); Idem, 16.iv.1975. J. Idrobo y C. Sáenz leg. (1 đ̊ ICN-MHN). Antioquia, Concepción x.1996 en rastrojo F. Serna y J.Hurtado leg. (1 † MEFLG); Alejandría xi.1974 A. Molina (1 † MEFLG). Bogotá D.C., Normandía. $2500 \mathrm{~m}$ 27.ii.1978. MLJM leg. (1 ふ̋ ICN-MHN). Caldas cañaveral, la mula $260 \mathrm{~m}$ i.2002 E. Martínez leg. (2 q MEFLG). Chocó, bahía Solano, Sector Huina, 26-27.viii.2006 E. Amat leg. (3 ð 5 ㅇ IAVH). Cundinamarca, Guayabetal. 12. ix.1976. G. Figueroa leg. (1 9 ICN-MHN); Mun. La Mesa Vda. El Espino Carroña 0032 18.xii.2002 JJW leg. (1 Î IAVH); Idem, excepto: Carroña 0008 19.xii.2002 JJW leg. (1 † IAVH); Idem, excepto: Carroña 0031 18.xii.2002 M.A.A.L leg. (1 $q$ IAVH). Fusagasuga. 12.x.1971. Sobre arbusto. H. Alarcón leg. (1 ^ UNAB); Idem, 12.x.1971. En arbusto. H. Alarcón leg. (1 q UNAB); Idem, excepto: 12.iv.1975. En maleza (1 o UNAB); Mesitas del Colegio. 01.xi.1973. Malezas. A. Villanueva leg. (1 §̂ UNAB); Arbeláez. 07.xi.1973. En maleza. Soleado. A. González leg. (1 O UNAB); Granada. 11.x.1999. A. Martínez y A. Rozo leg. (1 q UNAB). Huila, Neiva. 12.x.1974. Al vuelo. S. Mosquera leg. (1 + UNAB). Magdalena, PNN Tairona Gairaca Abanico Aluvial Estiércol 9.ii.1977 C. Kugler leg. (1 9 IAVH); Idem, excepto: sobre carne podrida 18.x.1976 C. Kugler leg. (3 ○ IAVH) . Meta, Villavicencio. 07.xi.1970. Gómez leg. (1 § ICN-MHN); Puerto Lleras. 27.v.1978. J. C. Uribe leg. (1 † UNAB); Idem. 27.v.1978. J. C. Uribe leg. (2 $q$ UNAB); Puerto Lopez, Pachiaquiaro, Finca El Saman 03.xi.2006 E. Amat y Z Reyes leg. (1 q 1 ふ IAVH). Tolima, Ambalema, Hacienda Buenavista de Caicedo. 22.viii.1977. M. Rodríguez leg. (1 + ICN-MHN). Vichada, Mun. Cumarimbo Cgto. Santa Rita PNN El Tuparro 5²1'48'N 6751'36”O 135m Bosque Inundable Trampa Exc. Humano \#6 30.I.2004 I. Quintero y E. González leg. (1 o IAVH); Idem, exepto:135m 5²1'20”N 6801'28”O Bosque de cerro Trampa de interceptación de vuelo \#T2 18-21.ii.2004 . (3 o IAVH); Idem, excepto: Trampa de interceptación de vuelo \#T2 18-21.ii.2004 (2 § IAVH).

\section{Compsomyiops Townsend}

Género neotropical fácilmente distinguible por presentar la parafacial setosa, característica un poco difícil de observar en algunos machos. Algunas especies fueron estudiadas detalladamente por Mello (1968) bajo el género Paralucilia. El género fue revisado por Dear (1985) quien reconoce 6 especies válidas. En el estudio de GonzalesMora et al,.. (1998) se sinonimiza C. boliviana (Mello, 1968) con C. arequipensis (Mello, 1968), los autores expresan la dificultad para encontrar diferencias significativas en las hembras y diferenciar las especies. Situación similar se presenta con las hembras revisadas en este trabajo. El macho de C. melloi Dear, 1985 es desconocido, por lo que su estado taxonómico aún no está claro y posiblemente en el futuro pueda cambiar; aquí se anota el registro para Bogotá de Dear (1985). En Colombia el género exhibe principalmente una distribución andina. No se presentan registros en otra región natural. La especie más común en Colombia es $C$. verena (Fig. 48) distribuida principalmente en la franja de los 1500 a 300 m de altura (Fig. 50). Esta mosca hemisinantrópica es fuertemente atraída por heces humanas, de allí su importancia sanitaria (Baugartner y Greenberg 1985); también se encuentra comúnmente sobre carroña. Ningún ejemplar de $C$. arequipensis se encontró de acuerdo con la descripción de Gonzales-Mora et al. (1998). Se sabe que tiende a ser hemisintantrópica y que se distribuye en altas localidades hasta los $3500 \mathrm{~m}$ (Baugartner y Greenberg 1985) de las montañas andinas en Bolivia, Ecuador, Perú y Colombia.

Material revisado. Compsomyiops verena (Walker, 1849): COLOMBIA. Antioquia, Medellín i.1976 E. Madrigal leg. (det Gagne) (1 + MEFLG); La Ceja x.1953 Gallego leg. (1 + MEFLG); Medellín 1950 Gallego leg. (1 9 MEFLG); Rionegro x.1946 Gallego leg. Silvestre (1 q MEFLG); Caldas Gallego, (1 q MEFLG); Guarne en vuelo i.1976 E. Madrigal en vuelo (1 9 MEFLG); Caldas ix.1973 R.Velez (1 + MEFLG); Antioquia. Río Claro. Reserva Ecológica El Cañón del río Claro. 01.x.1997. A. Alba leg. (1 đ̄ UNAB). Bogotá D.C. 2500 m 13.xi.1976. S. Zuluaga leg. (3 ๆ ICN-MHN); Bogotá D.C. 11.xi.1972 (1 o ICN-MHN); Normandía. 27.ii.1978. MLJM leg. (1 q ICN-MHN); Universidad Nacional de . 23.vi.1978. H. Torres leg. (1 q ICN-MHN); Idem, excepto: 19.iii.1974. J. Perea leg. (1 + ICN-MHN); Idem: excepto 24.ii.1980. J. Idrobo leg. (1 q ICN-MHN); Idem, excepto: $12 . i i .1982$ leg. (3 §̂ ICN-MHN); Idem, excepto: 10.ii.1982 leg. (1 స̃ ICN-MHN); Bogotá D.C. 10.x.1978. C. Gómez leg. (1 † ICN-MHN); Monserrate. 18.x.1970. H. Rojas leg. (1 q ICN-MHN); Alto del Cable. 3100 m 07.vii.1978. MLMJ leg. (1 O ICN-MHN); Idem, excepto: 30.vi.1978. MLJM leg. (11 § ICN-MHN); Idem, excepto: 07.vii.1978. MLJM leg. (1 §̊ ICN-MHN); Idem, excepto: 10.ii.1982 (1 సิ ICN-MHN); Bogotá D.C. 27.vi.1968. E. Ramírez leg. (1 § ICN-MHN). Idem, excepto: 06.vi.1968 (1 え ICNMHN). Bogotá D.C. 07.ii.1978. C. Hernandez leg. (1 $\lesssim$ ICN-MHN);Suba, "Lomas José". 24.iii.1974. J. Perea leg. (1 §̂ ICN-MHN); Bogotá D.C. 3.xii.1969. E. Barrera leg. (1 §̂ ICN-MHN); Bogotá ix.1961 G Paillier leg. (1 † MPUJ); Bogotá. 30.v.1970. Arbusto. Matallana leg. (2 స̃ UNAB); Bogotá. 14.iii.1968. Potrero. G. Arévalo leg. (1 đ̂ UNAB); Bogotá. 22.v.1969. Doméstica. E. Rabeya 
Clave para la identificación de especies del género Compsomyiops en Colombia.

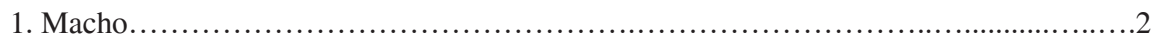

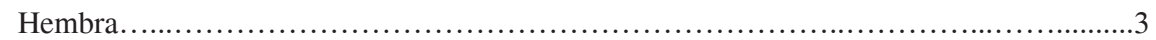

2. Ojos holópticos (Fig. 21), facetas agrandadas; fémures con manchas en su parte central y con los extremos de color castaño; basicosta amarilla a castaño, cercos y surstilos del macho en vista posterior como en la figura 33................. verena (Walker, 1849).

Ojos subholópticos (Fig. 23); la frente en su parte más estrecha con cada parafacial igual o ligeramente más ancha que el diámetro del ocelo anterior, facetas anteriores normales, fémures de coloración variable, cercos y surstilos del macho en vista posterior como en la

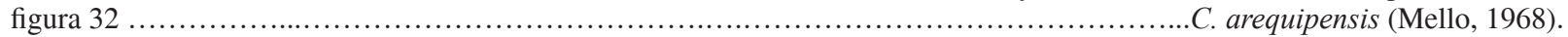

3. Tergos sin parches metálicos laterales........................................................................ melloi Dear, 1985.

Tergos con parches metálicos laterales que contrastan con la coloración metálica regular....4

4. Frente a la altura de la lúnula (Fig. 23) más estrecha que la placa frontoorbital, calípteros oscuros...

Frente a la altura de la lúnula (Fig. 23) igual o más ancha que la placa frontorbital, calípteros de coloración variable, manchas metálicas laterales presentes únicamente en el margen y en la superficie ventral del tercer al quinto

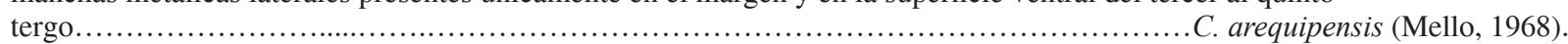

leg. (1 ð̊ UNAB); Bogotá. Sin fecha. En vuelo. C. Bonilla leg. (1 ð̂ UNAB); Bogotá. 18.ix.1970. Desperdicios. E. Benítez leg. (1 đ̃ UNAB); Bogotá. 10.vii.1970. En casa. M. Romero leg. (1 đ̃ UNAB); Bogotá. 17.ix.1974. En basura. M. Mejia leg. (1 q UNAB); Bogotá D.C., Parque Nacional, Estación de Carabineros. 11.xii.2002. L. Latorre y S. Jiménez leg. ( 2 2 INML-CF); Idem, 11.xii.2002 leg. (1 † 2 ते INML-CF); Idem, excepto: 02.xii.2002 leg. (1 q INML-CF); Idem, excepto: 3.xii.2002 leg. (1 $q$ INML-CF); Idem, excepto: 8.xii.2002 leg. (1 q INMLCF); Idm, excepto: 10.xii.2002 leg. (1 9 INML-CF); Idem, excepto: 26.xii.2002 leg. (1 9 INML-CF); Idem, excepto: 3.i.2003 leg. (1 O INML-CF); Idem, excepto: 01.xii.2002 leg. (1 § INML-CF); Idem, excepto: 19.xii.2002 leg. (1 స̂ INML-CF); Bogotá, 24.xii.2004. G. Camacho leg. (3 $\hat{\jmath}$ INML-CF); $1 \hat{\jmath}$, Instituto Nacional de Medicina Legal y Ciencias Forenses. G. Camacho leg. (1 $\widehat{\jmath}$ INML-CF); Alto del Cable. 3100 m 30.vi.1978. MLMJ leg. (14 q ICNMHN); Universidad Nacional de . ii.1982. J. Idrobo leg. (4 ㅇ ICN-MHN). Boyacá, SFF Iguaque Cabaña Chaina $5^{\circ} 25^{\prime} \mathrm{N} 73^{\circ} 27^{\prime} \mathrm{O} 2600 \mathrm{~m}$ Malaise 13.iv.5.v.2001 P. Reina Leg. M.1741 (1 † IAVH); Idem, excepto: 17.v.5.vi.2001. M.1768 (3 IAVH); Idem, excepto: 17.v.5.vi.2001. M.1768 (1 § IAVH); Idem, excepto: 31.viii.16.ix.2001A. Roberto leg. M.2066 (2 q IAVH); Idem, excepto: 31.viii.16.ix.2001.M.2066 (1 $\widehat{\jmath}$ IAVH); Idem, excepto: 2855m 6-23.v.2000. M.74 (1 § IAVH); Idem, excepto: 23.ix.11.x.2000 . M.752 (1 $\sigma^{\wedge}$ IAVH); SFF Iguaque La Planada $5^{\circ} 25^{\prime} \mathrm{N} 7^{\circ} 27^{\prime} \mathrm{O} 2850 \mathrm{~m}$ Malaise 11.x.1.xi.2000 P. Reina Leg. M.751 (2 ð IAVH); Sogamoso. 26.iii.1989. Basuras. G. Ardila leg. (1 † UNAB); Páez. 20.ii.1973. E. Cruz leg. (1 9 ICN-MHN). Cundinamarca, Zipaquirá, Planta de Tibitó. 17.ii.1973. A. Méndez leg. (5 O ICNMHN); Idem,. 17.ii.1973. A. Méndez leg. (1 ठ̀ ICNMHN); Mosquera, Corpoica-Tibaitatá. 4²4'N 74²12'O. 2543m. 27.vi.2001. G. Camacho y F. Ospina leg. (4 우 ICN-MHN); Idem, excepto: 26.vi.2001 (3 q ICN-MHN);
Idem, excepto: 28.vi.2001 (3 q ICN-MHN); Idem, excepto: 29.vi.2001 leg. ( 2 ICN-MHN); Idem, excepto: 25.vi.2001 leg. ( 1 ICN-MHN); La Calera. 2800 m 30.iv. 1978. MLMJ leg. (1 q ICN-MHN); Zipaquirá. 17.ii.1973. J. Rojas leg. (1 † ICN-MHN); Facatativa, Carretera San Francisco. 240 4.vii.1980. R. Viloria leg. (1 ô ICNMHN); Cundinamarca. 1496m. 8.x.1976. R. Gómez leg. (1 ð ICN-MHN); Fusagasuga. 4.iii.1971. En maleza. M. Delgadillo leg. (2 ð UNAB); Ubaté. 1.x.1994. A.Torres leg. (1 đ̃ UNAB); Ubaque. Vda. San Antonio. 4.x.1997. C. Bejarano, J. Díaz y E. Guzmán leg. (1 đ̊ UNAB); Soacha. 15.iv.1971. Al vuelo. R. Artunduaga leg. (1 † UNAB); Pacho. 30.xii.1971. G. Corredor leg. (1 q UNAB); Zipacón. 17.x.1993. A. Mora leg. (1 q UNAB); Caqueza. 3.iv.1994. J. Arias leg. (1 q UNAB); Simijaca. 02.x.1994. Fique y Reina leg. (1 $q$ UNAB). Magdalena, PNN Sierra Nevada de Santa Marta El Ramo 1048'N 73³9'O 2500 m Malaise 29.xii.2000 15.i.2001 J. Cantillo Leg. M.1184 (1 § IAVH). Meta, Villavicencio. 29.iii.1999. J. Arbelaez y F. Betancourt leg. (1 $\precsim$ UNAB); Idem, excepto: 22.v.1995. A. Perilla leg. (1 O UNAB); Puerto Porfia. 27.iii.1971. Al vuelo. E. Delgadillo leg. (1 9 UNAB); San Juan de Arama. 25.iii.1997. Ropain leg. ( 1 \&NAB). Norte de Santander, Cucuta. 05.iv.1969. Desperdicios. R. Lewis leg. (1 q UNAB). Quindio, Salento, La Montaña, CRQ. 05.ix.1998. C. Muñoz leg. (1 9 ICN-MHN). Tolima, Armero, Finca Inbora. 3.xi.1972. P. Carrillo leg. (1 9 ICN-MHN); Honda. 24.x.1970. Al vuelo. J. G. Zugo leg. (1 $\odot$ UNAB); Herveo. 26.xii.1971. Al vuelo. H. Quintero (1 † UNAB); Ibagué. 23.xii.1980. O. Jiménez leg. (1 đ̋ ICN-MHN).

\section{Chloroprocta Wulp}

Género monotípico representado por $C$. idioidea (Robineau-Desvoidy, 1830), distribuida desde el sur de los Estados Unidos y norte de México hasta Paraguay. En Colombia se ha registrado en los departamentos de 
Amazonas, Antioquia, Caldas, Meta, Risaralda y Vichada (Fig. 50) hasta el límite de los $2200 \mathrm{~m}$ de altura (Fig. 52). La especie se reconoce fácilmente por presentar la parafacial y el calíptero inferior desnudos y la cerda supra alar ausente, ô genitalia (cercos y surstilos) como la figura 34. Es una especie típica asinantrópica, fuertemente atraída por las trampas cebadas con pescado (Baugartner y Greenberg, 1985)

y una de las especies más abundantes de Calliphoridae en la selvas amazónicas del Brasil (Esposito, 1999); sus estados inmaduros se desconocen.

Material revisado. Chloroprocta idioidea (RobineauDesvoidy, 1830): COLOMBIA. Amazonas, río Igara Paraná, $15 \mathrm{~km}$ de la Chorrera. iv.1975. J. Idrobo y C. Saenz leg. (1 $\bigcirc$ ICN MHN); Caldas, Cañaveral La Mula 345m i.2002 rastrojo E. Martínez, (1 ํ MEFLG); Meta, PNN Sumapaz Porvenir Marayal 348'N 7349'O 535m Malaise 3-30.iv.2002 H. Vargas Leg. M.3112 (3 §ै ICN MHN); Puerto López. Pachiaquiaro. Finca El Samán. 4'3'37' 'N73'10'0''O. colecta manual.fecha03.xi.2006 E. Amat y Z Reyes leg. (3 ô 7 IAVH Risaralda, Pereira Vereda La Suiza SFF Otún Quimbaya. 1920 m 29.iv.2003 E. Manzano leg. (1 † MPUJ). Vichada, PNN El Tuparro Cerro Tomás 5²1'N 67²51'O 140 m Malaise 29.vii.8.viii.2000 W. Villalba leg. (2 9 IAVH); Idem, $250 \mathrm{~m}$ Malaise 22.v.3.vi.2001I. Gil leg. (1 q IAVH); Cumarimbo Cgto. Santa Rita PNN El Tuparro 5'18'27' 'N 67 57'00' 'O 35m Bosque Tierra Firme, trampa excremento humano \#123 14-16.ii.2004 I. Quintero y E. Gonzales leg. (1 9 IAVH).

\section{Paralucilia Brauer y Bergenstamm}

Algunas especies del género Paralucilia Brauer y Bergenstamm 1891 fueron estudiadas usualmente bajo el género Myiolucilia Hall, 1945; sin embargo, después del trabajo de Dear (1985) se sinonimizan los géneros y se agrupan todas en Paralucilia. A partir de ese momento se reportan nuevas sinonimias y nuevos registros en el estudio de Mariluis et al., (1994). En Colombia se conocían sólo 2 especies. Una es P. paraensis Mello, 1969 (= P. adespota sensu Dear, 1985) con un registro en el departamento de Cundinamarca y otro con localidad desconocida hecho por Dear (1985) en "Condota" sin datos sobre el departamento. La otra especie es P. fulvinota (Bigot, 1877), típica especie asinantrópica, distribuida desde México hasta Brasil con registro en el departamento de Cundinamarca (Fig. 50). En este estudio se registra por primera vez $P$. pseudolyrcea (Mello, 1969), especie asinantrópica de amplia distribución neotropical, que fue encontrada en zonas bajas de los valles interandinos. Aunque poco se sabe sobre su biología, algunos de sus caracteres diagnósticos para la identificación de las larvas fueron tratados por Trigo (2006).

Material revisado. Paralucilia paraensis (Mello, 1969): COLOMBIA. Cundinamarca Caqueza. 17.v.1997. X. Medina leg. (1 §o UNAB). Paralucilia fulvinota (Bigot, 1877): COLOMBIA. Bogotá. D.C., 15.xi.1970. Al vuelo. C. Herrera leg. (1 ¿̊ UNAB) Cundinamarca, Mun. La Mesa Vd. El Espino. En Carroña 16.xii.2002 M. Ariza leg. (2 9 IAVH). Paralucilia pseudolyrcea (Mello, 1969): COLOMBIA. Caldas, Cañaveral La Mula 235m-380 m i.2002 En rastrojo y bosque secundario E. Martínez leg. (20 ㅇ 18 ` MEFLG). Norcasia 5²1'80”N 7452'51'O 08.xi.2006. F. Forero leg. (1 $\odot 1$ गे IAvH).

\section{Género Hemilucilia Brauer}

Género endémico neotropical, con 7 especies, de las cuales 5 se registran en Colombia. Al parecer $H$. segmentaria (Fabricius, 1805) y H. semidiaphana (Rondani, 1850) son las especies más comunes en Colombia, se encuentran principalmente en zonas bajas aunque algunas especies

Clave para la identificación de especies de Paralucilia de Colombia.

1. Mesonoto (Fig. 2) brillante de color púrpura azul o verde metálico, prescutum sin bandas, macho con la parafacial y placas orbitales oscuras sin reflejos brillantes, cerdas postgenales (barba) y cabeza de color amarillo plateado o crema, espiráculo anterior (Fig. 1) usualmente castaño, calíptero superior desnudo, porción posterior del calíptero superior que se une con el calíptero inferior de coloración blanca, cercos y surstilos del macho en vista posterior como en la figura

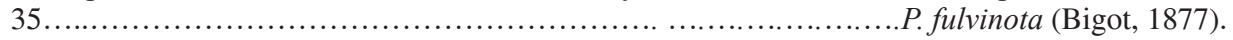

Mesonoto (Fig. 2) azul púrpura o verde con poco brillo, visto oblicuamente desde atrás con 3 bandas que alcanzan el escutelo (las laterales más conspicuas), cerdas postgenales (barba) de color dorado a amarillo naranja, espiráculo anterior (Fig. 1) amarillo o blanco, calíptero superior con o sin pelos en la superficie dorsal y de coloración variable.....................2

2. Espiráculo anterior amarillo o naranja, tibia posterior con 1 cerda posterodorsal, partes anteriores de los calípteros oscuras; macho con las placas frontorbitales con reflejos negros, brillantes alrededor de las cerdas frontales y sétulas negras por fuera de la fila frontral; superficie dorsal del calíptero superior con finos pelos, cercos y surstilos del macho en vista posterior como en la figura

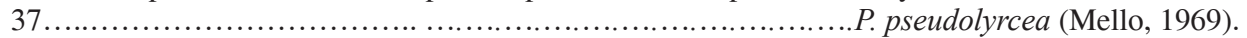

Espiráculo anterior blanco y expandido; tibia posterior con 2 cerdas posterodorsales, partes anteriores de los calípteros blancas; macho con las placas frontoorbitales sin reflejos oscuros y con vellosidad pálida fuera de la fila frontal, cercos y surstilos del macho en vista posterior como en la figura 36 .................................... paraensis (Mello, 1969). 
pueden llegar hasta los $1200 \mathrm{~m}$ (Fig. 52). Hemilucilia segmentaria (Fabricius, 1805) se encuentra ampliamente distribuida en Colombia mientras que $H$. semidiaphana (Rondani, 1850) se encontró comúnmente asociada con la región andina montañosa (Fig. 47). Poco se conoce sobre su biología y comportamiento reproductivo; exhiben una notable preferencia por trampas cebadas con pescado (Baugartner y Greenberg, 1985). Vale la pena destacar el primer registro de $H$. townsendi Shannon, 1926 para Colombia en la sierra nevada de Santa Marta, esta especie se conocía unicamente en Perú. Hemilucilia melusina Dear, 1985 es una especie asinantrópica rara, atraída principalmente por trampas cebadas con pescado (Baugartner y Greenberg, 1985), previamente registrada para la región andina montañosa en Perú y Colombia, aquí se registra un solo ejemplar proveniente de una reserva natural en relativo buen estado de conservación.

Material revisado. Hemilucilia melusina Dear, 1985: COLOMBIA. Risaralda, SFF Otún Quimbaya El Molinillo 443'N 75³4'O 2220 m Malaise 17.ii.4.iii.2003 G. López Leg. M.3696 (1 q IAVH). Hemilucilia segmentaria (Fabricius, 1805): COLOMBIA. Amazonas, PNN Amacayacu Matamata m1 3²41'S 70¹5'O $150 \mathrm{~m}$ Malaise 2 8-12.iii.2000 M. Sharkey y B. Brown leg. (1 q IAVH). Caldas, Cañaveral la Mula 260 m-380 m i.2002 En rastrojo y bosque secundario E. Martínez leg. (13 $q$ 3 đ̄ MEFLG). Chocó, PNN Utría Río San Pichí 6º1’N 77²0'O 10 m Malaise 30.vi.4.vii.2000 B. Brown leg. (1 q IAVH). Cundinamarca, Villeta. 14.iv.1996. P. García leg. (1 9 UNAB); La Mesa. Vda. San Javier. 11.ix.2003. Zona semi-urbana. En cultivo de mango. Al vuelo. 1196m. J. Pardo leg. (1 9 UNAB). Magdalena, PNN Tairona Los Naranjos 29.ix.1977 C. Kluger leg. (1 $\subsetneq$ IAVH); Santa Marta PNN Sierra Nevada de Santa Marta , C Minca(3 $\delta$ IAVH) . Meta, Macarena. 24.iii.1999. C. Santana leg. (1 q UNAB). Putumayo, PNN La Paya Fca. Charapa 08'S 7457'O 330 m Malaise 26.iii.10.iv.2002 R. Cobete leg. (1 9 IAVH). Valle del Cauca, Jardín Botánico José María Céspedes 1100 m 9.x.1998 pitfall P.Cogua y Romero leg. (2 9 MPUJ). Hemilucilia semidiaphana (Rondani, 1850): COLOMBIA. Caldas, Cañaveral la Mula 260 m-380 m i.2002 En rastrojo y bosque secundario E. Martínez leg. (29 911 o MEFLG). Cundinamarca, Mun. La Vega vereda El Espino Sobre Carroña M32 9.xii.2002 JJW leg. (1 ๆ IAVH); Idem, excepto: M32 18.xii.2002 JJW leg. (1 † IAVH); Pacho. 14.x.1998. L. Gallego leg. (1 §̂ UNAB). Tolima, Armero, Cambao, Cerro de Santo Tomas $250 \mathrm{~m}$ 12.xi van sorem (2 + MPUJ). Valle del Cauca, Jardín Botánico José María Céspedes 1100 m 9.x.1998 pitfall P Cogua y Romero leg. (2 q MPUJ). Hemilucilia townsendi Shannon, 1926: COLOMBIA. Magdalena, Santa Marta PNN Sierra nevada de Santa Marta C. minca Cuenca Rio

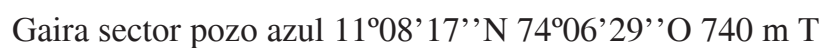
exc humano T163 9-19.x.2004 H. Garcia y D. Ospino leg. (1 $q$ IAVH).

Clave para la identificación de especies del género Hemilucilia de Colombia.

1. Superficie ventral de la vena costal setulosa hasta la ruptura subcostal; macho sin cerda frontal reclinada; hembra con débiles cerdas frontorbitales cuyo ápice no alcanza el nivel de la lúnula. 2

Superficie ventral de la vena costal setulosa hasta la unión con R1 o más allá; macho con la cerda superior frontal reclinada; hembra con fuertes cerdas orbitales cuyos ápices alcanzan y sobrepasan el nivel de la lúnula....................

2. Base del ala fuertemente ahumada (celdas bc, c, sc, $1 / 4$ de la 1 , primer tercio de la br, b-m y cup); calípteros negros o castaño oscuro y opacos, tórax y abdomen completamente oscuros, superficie dorsal del calíptero superior sin vellosidades; cercos y surstilos

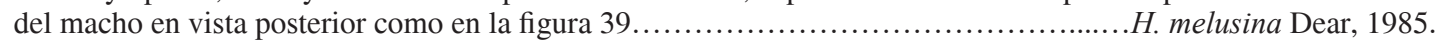

Base del ala levemente ahumada o hialina; calípteros claros o levemente castaños, superficie dorsal del calíptero superior usualmente con vellosidades....

3. Espiráculo posterior castaño oscuro; área anterior y lateral del tórax del mismo color metálico; superficie dorsal del calíptero superior setulosa en ambos sexos; cercos y surstilos del macho en vista posterior como en la figura 41.

..H. semidiaphana (Rondani, 1850).

Espiráculo posterior amarillo o crema; área anterior y lateral del tórax amarilla y/o naranja, anepisterno usualmente amarillo; superficie dorsal del calíptero superior desnuda en los machos y setulosa en la hembra; cercos y surstilos en vista posterior, como en

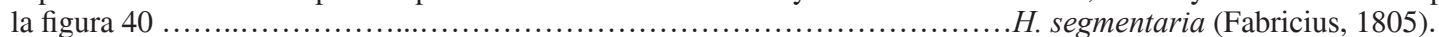

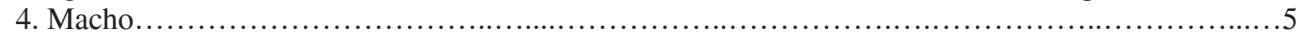

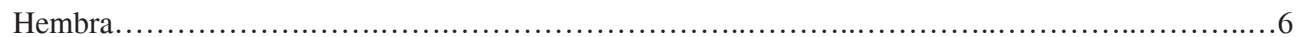

5. La región más estrecha de la frente, más amplia que el ancho del triángulo ocelar; cercos y surstilos del macho en vista posterior como en la figura 38 .

...H. benoisti Séguy, 1925. (Macho desconocido) H. townsendi Shannon, 1926.

6. Proepisterno y lóbulos postpronotales (Fig. 1) total o parcialmente amarillos ..H. townsendi Shannon, 1926.

Proepisterno y lóbulos postpronotales (Fig. 1) azules o verdes con brillo metálico .H. benoisti Séguy, 1925 . 


\section{Subfamilia Toxotarsinae}

Endémica de la región Neotropical, para Colombia se registran Sarconesiopsis magellanica (Le Guillou, 1842), Chlorobrachycoma splendida Townsend, 1918 y Roraimomusca roraima Townsend, 1935. La subfamilia fue revisada por Dear (1979), sus especies son fácilmente reconocibles por poseer vellosidades en la superficie ventral de la vena troncal o vena remigio, característica poco común entre los califóridos. Varios autores concuerdan con la extrema dificultad para diferenciar y delimitar sus géneros. En Perú, S. magellanica (Le Guillou, 1842) resultó ser una de las especies con importancia medica más relevante, típica eusinantrópica, regularmente se encuentra en casas y edificios (Baumgartner y Greenberg. 1985). En Colombia se encontró sobre la cordillera oriental, en el rango de los 1200 hasta los $3100 \mathrm{~m}$ de altitud (Fig. 51-52), y por su característica endófila y eusinantrópica representa un gran potencial en el uso como indicador forense. C. splendida Townsend, 1918 es una especie típica hemisinantrópica de altas localidades y dominante desde los $2800 \mathrm{~m}$ (Baumgartner y Greenberg. 1985) en Colombia se registró desde los $2500 \mathrm{~m}$ hasta los $3500 \mathrm{~m}$ de altura (Figs. 51$52)$. $R$. roraima Townsend, 1935 habita altas elevaciones de los Andes venezolanos, colombianos y bolivianos, Dear (1979) registra un macho de esta especie para Colombia con los siguientes datos "Cerro Quemado, San Lorenzo Mt., 17. xii. 1972, 1 ,CAS.” depositado en la California Academy of Sciences, San Francisco, aunque existen varias localidades con el mismo nombre. Posiblemente este registro corresponda con alguna localizada en el pie de monte de la sierra nevada de Santa Marta, comprendida entre el cerro quemado y la cuchilla de San Lorenzo en el municipio de Santa Marta, departamento de Magdalena.

Material revisado. Chlorobrachycoma splendida Townsend, 1918: COLOMBIA. Bogotá D.C, Humedal de la Conejera M 83 Muestreo Acueducto E. Blanco leg. (1 q IAVH); Transv 66 N 145-4 52540 m Colecta Manual 3.iv.2005 J.Villa leg. (4 + IAVH).Cundinamarca, PNN Sumapaz Bocatoma. Cerro el Zapato $4^{\circ} 14^{\prime} \mathrm{N} 74^{\circ} 12^{\prime} \mathrm{O}$ 3560 m Pantrap 6-7.xi.2002 A. Patiño, H. Ángel y D.
Arias leg. (1 o IAVH); Idem, Malaise 2-17.i.2003 A. Patiño leg. (3 † IAVH). Sarconesiopsis magellanica (Le Guillou, 1842): COLOMBIA. Antioquia, Guarne $2280 \mathrm{~m}$ i.1976 E. Madrigal en Maleza (det Gagne. viii.1977) (4 $q$ MEFLG). ). Bogotá D.C,. 18.ix.1977. Alba leg. (2 q ICN MHN); Universidad Nacional de . 02.ii.1973. P. Carrillo leg. (1 q ICN MHN); Marsella. 3.vi.1974. L. Barbosa leg. (1 q ICN MHN); Bogotá D.C. 20.x.1976. J. Martínez leg. (1 o ICN MHN); Alto del Cable. 3100 m 30.vi.1978. MLMJ leg. (1 9 ICN MHN); Idem, excepto: 7.viii.1978 (1 § ICN MHN); Normandía. 2500 m 27.ii.1978. MLMJ leg. (1 §̃ ICN MHN); Bogotá. 08.xi.1970. G. Izquierdo leg. (1 q UNAB); Bogotá ix.1961 G Jaillier leg. (10 q MPUJ); Subía. 15.x.1999. A. Martínez y A. Rozo leg. (1 q UNAB). 1 Bogotá. 30.xi.1996. Marleny C. leg. (1 q UNAB); Bogotá D.C. 18.iv.1976. R. Gómez leg. (1 đ̃ ICN MHN); Bogotá. 9.iv.1995. L. Palacios leg. (1 우 UNAB); Bogotá. 09.xii.1973. Al vuelo. N. Torres leg. (1 q UNAB); Bogotá. 28.xi.1974. Al vuelo. J. Garzón leg. (1 q UNAB). Boyacá, SFF Iguaque Cabaña Carrizal 5²5’ N $73^{\circ} 27^{\prime} \mathrm{O} 2850 \mathrm{~m}$ Malaise 27.iii.16.iv.2001 P. Reina leg. (1 † IAVH); Idem, excepto: $2600 \mathrm{~m} \mathrm{13.iv.5.v.2001} \mathrm{(3}$ q IAVH); Idem, excepto: 17.v.5.vi.2001 (1 q IAVH). Cundinamarca, Mosquera, Corpoica-Tibaitatá. 4º42'N 74º'12'O. 2543m. 22.vi.2001. G. Camacho y F. Ospina leg. (3 9 ICN MHN); Idem, excepto: 25.vi.2001 (3 9 ICN MHN); ); Idem, excepto: 21.vi.2001 (1 q ICN MHN); Idem, excepto: 26.vi.2001 (1 9 ICN MHN); Idem, excepto: 23.vi.2001 (2 ð ICN MHN); Idem, excepto: 16.vi.2001 (2 ๙ ICN MHN); Idem, excepto: 28.vi.2001 (1 §ิ ICN MHN); Idem, excepto: 29.vi.2001 (1 $ぇ$ ICN MHN); Idem, excepto: 25.vi.2001 (1 § ICN MHN) Zipaquirá. 10.viii.1970. H. García leg. (1 9 ICN MHN); Idem, excepto: 04.x.1970 (1 † ICN MHN); Idem, excepto: 15.vi.1970 (1 q ICN MHN); Río de Bogotá. xi.1969. Socorro leg. (1 q ICN MHN); Sasaima. 1191m. 05.iii.1976. M. Leó. leg. (1 §̋ ICN MHN); La Calera. 2800 m 30.iv.1978. MLMJ leg. (1 §ิ ICN MHN); Cajica 2650 m 7.xii.1940 (Det Hall. 1941) (1 q MEFLG); Anapoima. 16.xi.1989. L. Narváez leg. (1 † UNAB); Zipaquirá. 3.xi.1989. Hojas. N. Acero leg. (1 9 UNAB); Fusagasugà. 15.xi.1970. Al vuelo. I. A.

Clave para las especies de Toxotarsinae de Colombia.

1. Ojos densamente poblados con finas vellosidades doradas......Roraimomusca roraima Townsend, 1935.

Ojos glabros, sin finas vellosidades................................................2

2. Calíptero inferior intensamente ahumado; vellosidades de la arista alcanzan la mitad o algo más de su longitud; la longitud de los pelos más largos de la arista sobrepasa el ancho del flagelómero I; cercos y surstilos del macho en vista posterior como en la figura 43 Sarconesiopsis magellanica (Le Guillou, 1842).

Calíptero inferior blanco o amarillento; vellosidades de la arista no alcanzan la mitad de su longitud; la longitud de los pelos más

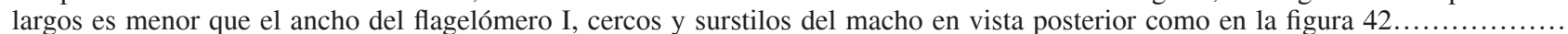
Chlorobrachycoma splendida Townsend, 1918. 
Pabón leg. (1 q UNAB); Sopó. 11.iv.1999. J. Sierra leg. (1 † UNAB); Cajicá. 07.iii.1921. Al vuelo. L. Venegas leg. (3 ๆ UNAB); Guaduas. 20.x.1971. L. H. García leg. (1 q UNAB); Silvania. 12.vi.1971. S. Barrera leg. (1 우 UNAB); La Mesa. 18.iv.2002. 1200 m En Solanaceae. W. Villamil leg. (1 q UNAB); Chocontá. 21.x.1970. E. Molina leg (1 đ̃ UNAB); Albán. 07. .1996. J. Castillo leg. (1 ð̃ UNAB); Vergara.13.vi.1970. C. Pardo leg. (1 đo UNAB); Ubaqué. Vda. San Antonio. 4.x.1997. Hoja de rosa, soleado. C. Bejarano, J. Díaz y E. Guzmán leg. (1 §̋ UNAB); Magdalena, PNN Sierra Nevada de Santa Marta El Ramo 1048'N 73³9'O 2500 m Malaise 29.xii.2000 15.i.2001 J. Cantillo leg. (1 § IAVH). Nariño Pasto 2600 m 10.xii.2007 D. Granja leg. (3 స̃ IAVH) Santander, Barbosa. 7.iv.1971. Al vuelo. P. Cadena leg. (1 $q$ UNAB). Valle del Cauca, Cali, Farallones. 4.v.1993. G. Andrade leg. (1 + ICN MHN)

\section{Agradecimientos}

Al proyecto Diversidad de Insectos de Colombia, desarrollado por el Instituto Alexander von Humboldt (IAvH) y patrocinado por la National Science Foundation (NSF) DEB 9972024 y 0205982, por el cual fue posible este trabajo. A Jon K. Gelhaus de la Academia de Ciencias de Filadelfia, a Michael Sharkey de la Universidad de Kentucky y a mi actual tutor, Jose Albertino Rafael, por su apoyo incondicional. A los curadores y personas encargadas de las colecciones visitadas; en Villa de Leyva, a Claudia Medina, Diego Perico, Miguel Ángel Torres, Edwin Torres y Zenaida Reyes; en el Instituto de Ciencias Naturales de la Universidad Nacional de Colombia-Bogotá, a Germán Amat. En la Facultad de Agronomía de la Universidad Nacional de Colombia-Bogotá, a Javier Martínez. Al Museo Francisco Luis Gallego de la Universidad Nacional Sede Medellín y en particular a Jhon Albeiro Quiroz "Black". En la Universidad Javeriana de Bogotá, a Giovanni Fagua. En el Instituto de Ciencias Forenses y Medicina Legal en Bogotá, a Ginna Camacho. También a Sohath Yussef por sus sugerencias al manuscrito. De manera muy especial a mi familia, Domingo, Cecilia, Germán y Zoila, por el apoyo incondicional a mi quehacer científico.

\section{Literatura citada}

Amat, E., M. C. Vélez y M. Wolff. 2008 Clave ilustrada para la identificación de los géneros y las especies de califóridos (Diptera: Calliphoridae) de Colombia. Caldasia 30:231-244.

Amorim, D. S., C. Silva y M. I. Balbi. 2002. Estado do conhecimento dos díptera neotropicais. In Proyecto de Red Iberomaericana de Biogeografía y Entomología Sistemática,
Pribes 2002, C. Costa, S. A.Vanin, J. M. Lobo y A. Melic (eds.). Monografías tercer milenio, vol 2. Sociedad Entomológica Aragonesa (SEA) y Cyted, Zaragoza. 29-36.

Barreto, M., M. E. Burbano y P. Barreto. 2002. Flies (Calliphoridae, Muscidae) and beetles (Silphidae) from human cadavers in Cali, Colombia. Memórias do Instituto Oswaldo Cruz 97:137-138.

Baumgartner, D. L. 1988. Spread of introduced Chrysomya blowflies (Diptera, Calliphoridae) in the neotropics with records new to Venezuela. Biotropica 20:167-168.

Baumgartner, D. y B. Greenberg. 1984. The genus Chrysomya (Diptera: Calliphoridae) in the New World. Journal of Medical Entomology 21:105-113.

Baumgartner, D. y B. Greenberg. 1985. Distribution and medical ecology of the blow flies (Díptera: Calliphoridae) of Peru. Annals of the Entomological Society of America 78:565587.

Carvalho, C. J. B de. y P. B. Ribeiro. 2000. Chave de identificaçăo das especies de Calliphoridae (Diptera) do sul do Brasil. Revista Brasileira de Parasitologia Veterinária 9:169-173.

Carvalho, C. J. B de. y C. A.de Mello Patiu. 2008. Key to the adults of the most common forensic species of Diptera in South America. Revista Brasileira de Entomologia 52:390406

Dear, J. 1979. A revision of the Toxotarsinae (Diptera: Calliphoridae) Papéis Avulsos de Zoologia 32:145-182

Dear, J. 1985. A revision of the new world Chrysomyini (Diptera: Calliphoridae) Revista Brasileira de Zoologia 3:109-169.

Esposito, M. C. 1999. A fauna de moscas varejeiras (Diptera: Calliphoridae) da Amazônia e sua ecologia na região de Caixuanã e cidade de portel/Estado de Pará. Tese para obtenção do titulo de doutor, Instituto Nacional de Pesquisas da Amazônia. Manaus, Amazonas. 133 p.

Florez , E. y M. Wolff. 2009. Descripción y clave de los estadios inmaduros de las principales especies de Calliphoridae (Diptera) de importancia forense en Colombia. Neotropical Entomology 38:418-429.

Gonzales-M. D., Peris S. V. y J. C. Mariluis. 1998. Notas sobre la taxonomía y distribución del género Compsomyiops Townsend, 1918 (Diptera, Calliphoridae). Boletín de la Real Sociedad Española de Historia Natural (Sección Biológica) 94:15-21.

Guimarães, J. H. 1977. A systematic revision of the Mesembrinellidae, stat. nov. (Diptera, Cyclorrhapha). Arquivos de Zoologia 29:1-109.

Guimarães J.H. 1979. Dispersal and distribution of the three newly introduced species of Chrysomya Robineau-Desvoidy in Brasil (Diptera: Calliphoridae). Revista Brasileira de Entomologia 23: 245-255.

Guimarães, J. H., N. Papavero y A. P. Prado. 1983. As Miíases na Região Neotropical. Revista Brasileira de Zoologia 1:239416.

Guimarães, J. H., A. P. Prado y A. P. Linhares. 1978. Tree newly introduced blowfly especies in southern Brazil (Diptera, Calliphoridae). Revista Brasileira de Entomologia 22:53-60.

Greenberg, B. y M.L. Szyska. 1984. Immature stages and biology of fifteen species of Peruvian Calliphoridae (Diptera). Annals of the Entomological Society of America 77:488-517. 
Hall, D. G. 1948. The blowflies of North America, vol 4. Thomas Say Foundation, Entomological Society of America, Lafayette, Indiana. 477 p.

Hijmans, R. J., M. Cruz, E. Rojas y L. Guarino 2001 DIVAGIS, version 5.4. A geographic information system for the management and analysis of genetic resources data. Manual. International Potato Center and International Plant Genetic Resources Institute, Lima, Peru. Available: http://www.divagis.org/; consulta: 17.11.09.

James, M. T. 1970. Family Calliphoridae, Fas. 102. In A catalogue of the America South of the United States. Museu de Zoologia da Universidade de São Paulo 102: 28 p.

Jiron, L. F. 1979. Sobre moscas califóridas de Costa Rica (Diptera; Cyclorrapha) Brenesia 16: 221-222.

Lopes, H. S. y D.O. Albuquerque. 1982. Notes on Neotropical Calliphoridae (Diptera) Revista Brasileira de Biologia 42: 63-69.

Mariluis, J. C. 1980. Presencia del género Chrysomya Robienau y Desvoidy 1830 en la región neotropical (Calliphoridae, Chrysomyiinae, Chrysomyiinii). Revista de la Sociedad Entomológica Argentina 39:126.

Mariluis, J. C. 1981. Clave para la identificación de los Calliphoridae de la República Argentina (Diptera). Revista de la Sociedad Entomológica Argentina 40: 27-30.

Mariluis, J. C. 1983. Presencia del género Chrysomya en la región neotropical (Calliphoridae, Chrysomyiinae, Chrysomyiiniii). Revista de la Sociedad Entomológica Argentina 42:141142.

Mariluis J. C. y S. V. Peris. 1984. Datos para una sinopsis de los Calliphoridae neotropicales. EOS- Revista Española de Entomología 40:67-86.

Mariluis, J. C. y J. A. Schnack. 2001. Calliphoridae de la Argentina. Sistemática, ecología e importancia sanitara (Diptera, Insecta). In Actualizaciones en artropodología sanitaria argentina, O. D. Salomon (ed.). Serie Enfermedades Transmisibles, Publicación Monográfica 2. Fundación Mundo Sano, Buenos Aires, Argentina. p.23-27.

Mariluis, J. C. y P. R. Mulieri. 2003. The distribution of the Calliphoridae in Argentina (Diptera). Revista de la Sociedad Entomológica Argentina 62:85-97.

Mariluis, J, C., M. D. Gonzales y S. V.Peris. 1994. Consideraciones sobre el género Paralucilia Brauer et Bergenstamm, 1891 (Diptera, Calliphoridae). Boletín de la Real Sociedad Española de Historia Natural (Sección Biológica) 91:15-18.

Mavárez, M., A. Espina., F. Barrios. y J. L. Fereira. 2005. The forensic entomology and the neotropic. Cuadernos de Medicina Forense 11:23-33.

McAlpine, J. F. 1981. Morphology and terminology, Monograph 27. In Manual of Neartic Diptera, vol. 1, J. F. McAlpine (ed.). Agriculture Canada, Ottawa. p. 9-63.

McAlpine J. F. 1989. Phylogeny and classification of the Muscomorpha, Monograph 32. In Manual of Nearctic Diptera, vol. 3, J. F. McAlpine (ed.). Research Branch, Agriculture Canada, Ottawa. p. 1397-1518.

Mello, R.P.de 1962. Contribuição ao estudo dos Mesembrinellinae sul americanos (Diptera, Calliphoridae) Studia Entomologica 10:1-80.

Mello, R.P. de 1968. Contribução ao estudo do gênero
"Paralucilia" Brauer y Bergenstamm, 1891 (Diptera, Calliphoridae) Revista Brasileira de Biologia 28:177-192.

Mello, R.P. de 2003. Chave para a identificaçăo das formas adultas das especies da familia Calliphoridae (Diptera, Brachycera, Cyclorrhapha) encontradas no Brasil. Entomologia y Vectores 10:255-268.

Paralupi, N. D. 1991. Calliphoridae (Diptera) de Manaus: Levantamento taxonómico, sazonalidade, atividade de vôo, desenvolvimento ovarino e potencialidade como vetores de baterías nas feiras libres. Tese, Instituto Nacional de Pesquisas da Amazônia, Manaus, Amazonas. 123 p.

Pape, T., M. Wolff y E. Amat. 2004. Los califóridos, sarcofágidos, éstridos y rinofóridos de Colombia. Biota Colombiana 5:201208.

Peris, S.V. 1985. Some additional data on the distribution of Chrysomya species in South America. EOS- Revista Española de Entomología 61: 239-240.

Peris, S. V. 1987. La invasión de las especies de Chrysomya en América (Dipt. Calliphoridae) Graellsia 43:205-210.

Peris, S. V. 1992. Claves preliminares para los géneros de las subfamilias Toxotarsinae, Chrysominae y Rhiniinae (Diptera: Calliphoridae) del mundo. Boletín de la Real Sociedad Española de Historia Natural (Sección Biológica) 88:79-98.

Peris, S.V. y D. Gonzales-Mora. 2005. Clave de identificación para los géneros de Calliphoridae del mundo. Subfamilias con vena remigium desnuda y creación de una nueva subfamilia. Boletín de la Real Sociedad Española de Historia Natural (Sección Biológica) 99:115-144.

Peris, S. V. y J. C. Mariluis. 1984. Notas sobre los Mesembrinellidae. Eos 60:251-265.

Povolny, D. 1971. Synanthropy. In Flies and disease, ecology, classification, and biotic associations, vol. 1, B. Greenberg (ed.). Princeton University Press, Princeton, New Jersey. p. 17-54.

Rognes, K. 1986. The systematic position of the genus Helicobosca Bezzy with a discussion of the monophilyly of the calyptrates fasmilies Calliphoridae, Rhinophoridae, Sarcophagidae and Tachinidae (Diptera). Entomologica Scandinavica 17:75-92.

Rognes, K. 1997. The Calliphoridae (Blowflies) (Diptera: Oestroidea) are not a monophyletic group. Cladistics 13:2766.

Rognes, K. y H. E. H. Paterson. 2005. Chrysomya chloropyga (Wiedemann, 1818) and C. putoria (Wiedemann, 1830) (Diptera: Calliphoridae) are two different species. African Entomology 13:49-70.

Shannon, R. C. 1926. Synopsis of the American Calliphoridae (Diptera). Proccedings of the Entomological Society of Washington 28:115-139.

Trigo, A.V. 2006. Descripción de las larvas II, III y el pupario de Compsomyiops

fulvicrura (Diptera: Calliphoridae). Revista de la Sociedad Entomológica Argentina 65: 87-99.

Wolff, M., A. Uribe, A. Ortiz y P. Duque. 2001. A preliminary study of forensic entomology in Medellín, Colombia. Forensic Science International 120: 53-59.

Zumpt, F. 1965. Myiasis in man and animals in the old world. Butterworths, London. 267 p. 

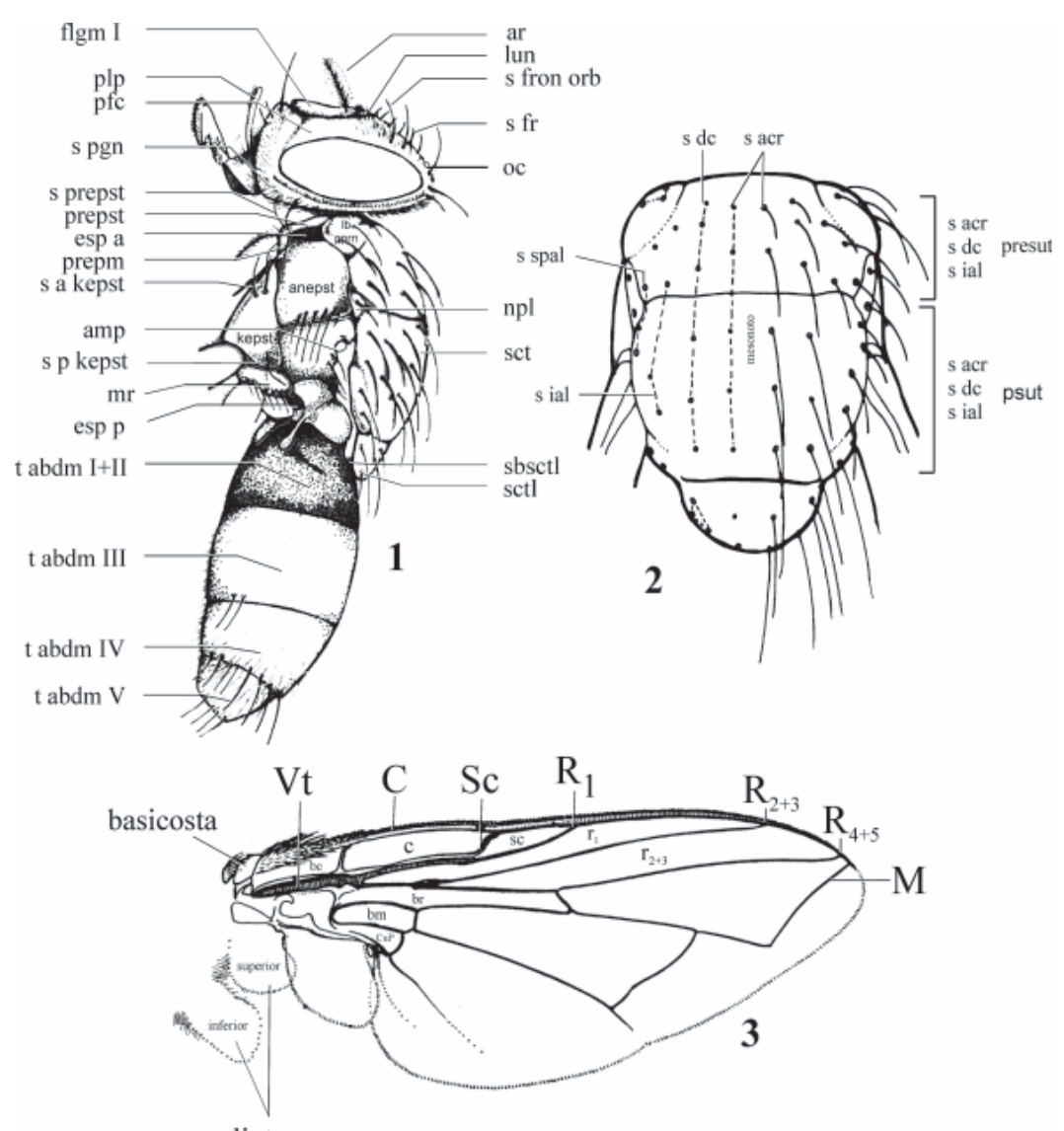

Figuras 1-3. 1. Morfología y plan corporal de una mosca califórida. amp, ampolla; anepst, anaepiesternon; ar, arista; esp a, espiráculo anterior; esp p, espiráculo posterior; fglm I; flagelomero 1; kepst, kataepiesternon; lb pprn, lóbulo pospronotal. lun; lunula; oc; ocelo; plp; palpos; pfc, parafacial; prepm, proepimeron; prepst, proepiesternon; s a kepst; cerda anterior kataepiesternal; s fr; cerdas frontales; s fron orb, cerdas frontorbitales; s prepst, cerda proepiesternal; s a kepst; cerda anterior kataepiesternal; s p kepst; cerda posterior kataepiesternal; s pgn; cerdas postgenales; sbsctl; subescutelo; sct; escutum; sctl; escutelo; t abdm; terguitos abdominales. 2. Quetotaxia toráxica de una mosca califorida; presut, presuturales; psut, postsuturales; $\mathrm{s}$ acr, cerdas acrosticales; s dc, cerdas dorsocentrales; s ia, cerdas intra alares; s spal, cerdas supra alares. 3. Venación típica de una mosca califorida. $\mathrm{C}$, vena costal; $\mathrm{M}$, vena media; $\mathrm{Vt}$, vena troncal (Remigio); R1, Vena radial 1; Sc, vena
Figuras 4-15. 4. Detalle subescutelo Mesembrinellinae. 5. Aleta dorsal del espiráculo posterior abierto, mr, meron. 6. Vena $M$ pronunciada. 7. Detalle subescutelo normal. 8 . Vena M angulosa. 9. Detalle de la vena troncal dorsalmente desnuda. 10. Ampolla ovoide con pilosidad corta. 11.Vena troncal setosa. 12. Ampolla en forma de riñón con pilosidad larga. 13. Detalle de la base de la vena $R$ ventralmente setosa. 14. Detalle del calíptero inferior con su borde anguloso y pilosidad típica de Paralucilia sp. 15. Calíptero divergente de la línea del escutelo.
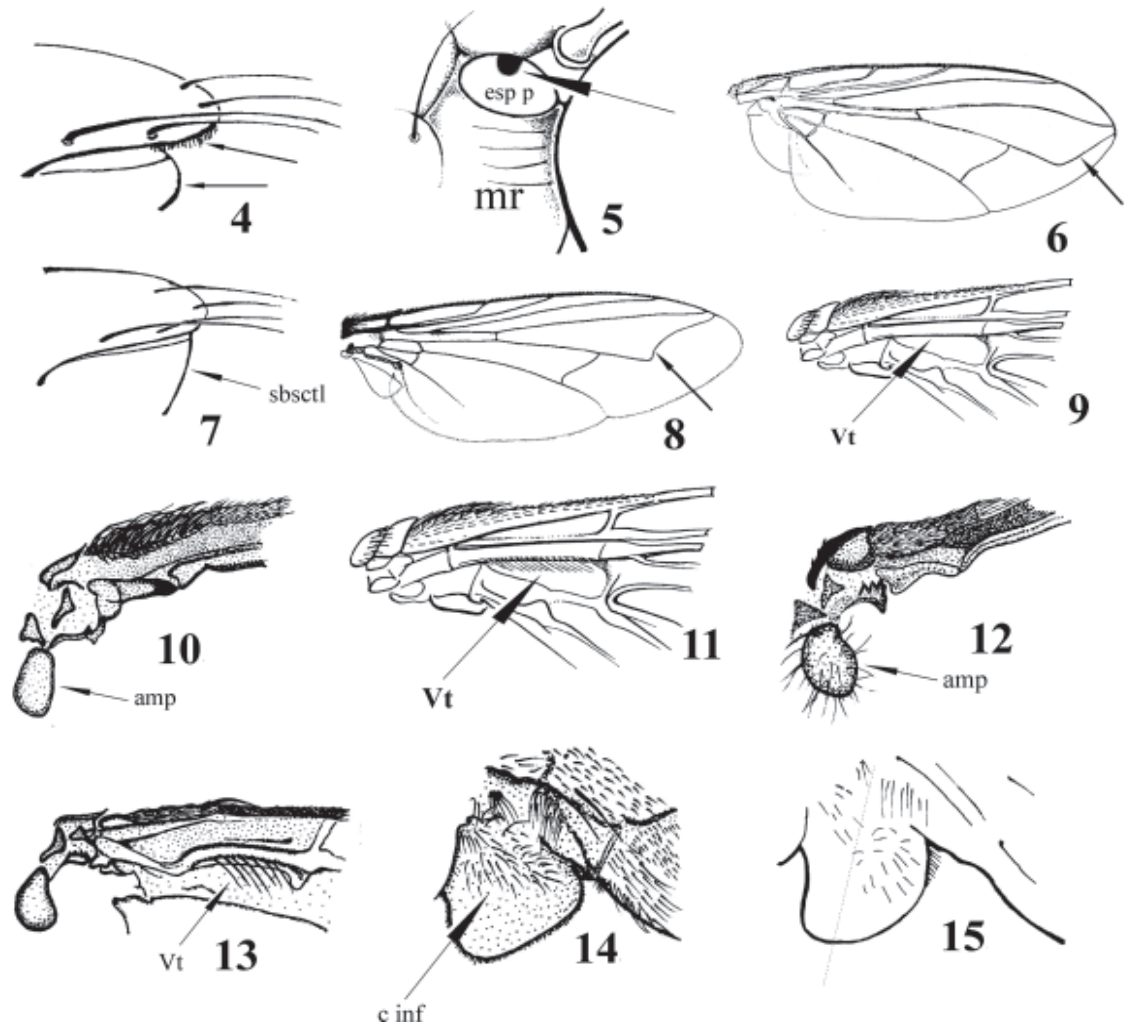


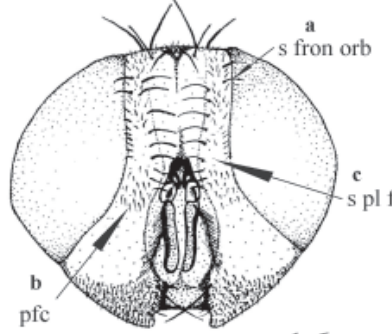

16

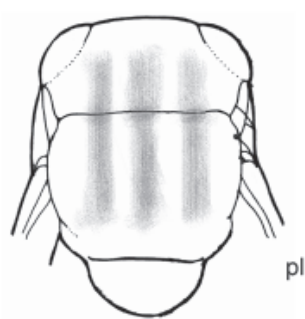

17

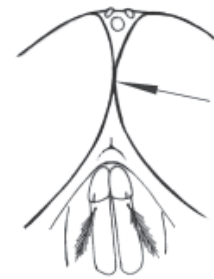

21

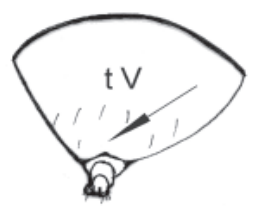

20

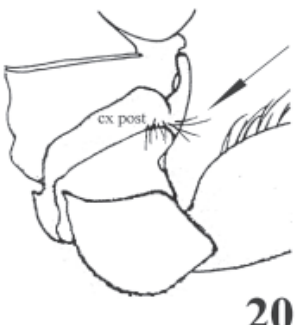

b)

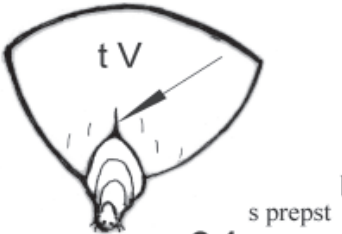

24

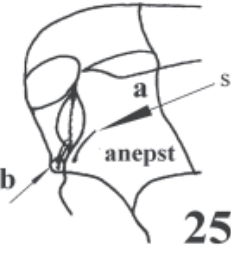

25
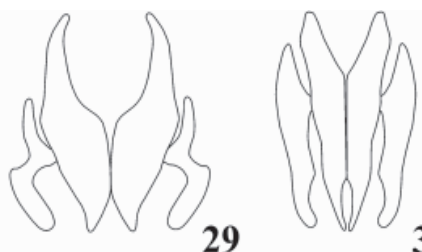

27

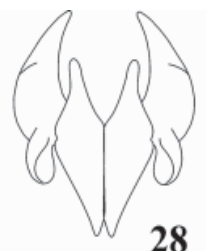

29
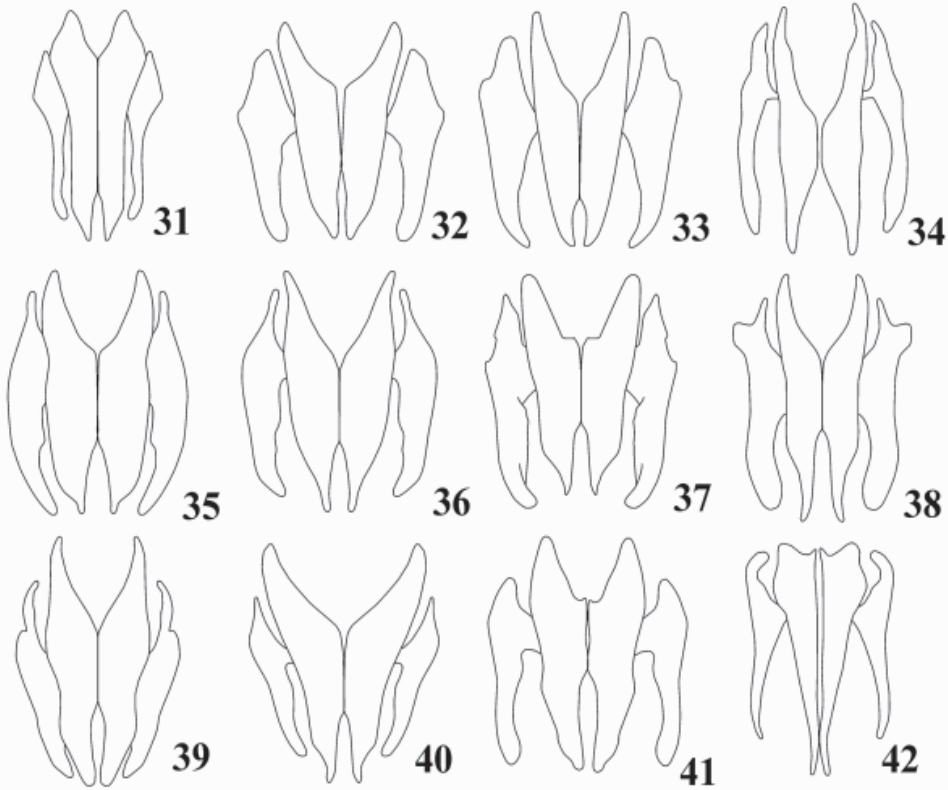

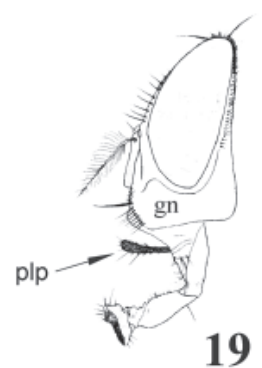

22

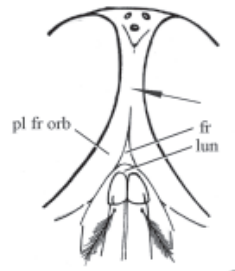

23

Figuras 16-26. 16. Detalles de la cabeza de una hembra: s fron orb, cerdas frontoorbitales lateralmente proyectadas (16a); pfc, parafacial setosa (16b); s pl f, cerdas de la placa frontal (16c). 17. Bandas longitudinales a la altura del mesonoto. 18. Detalle palpo filiforme. 19. Detalle palpo normal. 20. Detalle del borde superior de la coxa posterior en C. idioidea (modificada de Dear, 1985). 21. Condición holóptica en machos. 22. Tergo abdominal $\mathrm{V}$ en hembras sin incisión dorsal. 23. Condición subholóptica en machos: fr, frente; gn, gena: Lun, lúnula; pl fr orb, placa fronto orbital. 24. Tergo abdominal $\mathrm{V}$ en hembras con incisión dorsal. 25. Detalle del tórax en vista lateral, cerda estigmática presente (25a); cerdas proespiesternales (25b). 26. Detalle del tórax en vista lateral, cerda estigmática ausente (26a); cerdas proespiesternales (26b).

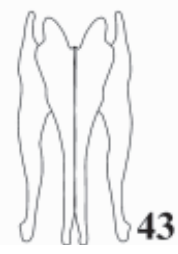

43

Figuras 27-43. Detalle de los genitalia de los machos (cercos y surestilos) en vista posterior. 27. Chrysomya albiceps. 28. Chrysomya megacephala. 29. Chrysomya putoria (modificada de Rognes y Patherson, 2005). 30. Cochliomyia hominivorax (modificada de Guimaraes et al., 1983). 31. Cochliomyia macellaria. 32. Compsomyiops arequipensis (modificada de Mello, 1968).33. Compsomyiops verena. 34. Chloroprocta idioidea. 35. Paralucilia fulvinota (modificada de Mello, 1968). 36. Paralucilia paraensis (modificada de Mello, 1968). 37. Paralucilia pseudolyrcea (Mello, 1969). 38. Hemilucilia benoisti. 39. Hemilucilia melusina. 40. Hemilucilia segmentaria. 41. Hemilucilia semidiaphana. 42. Chlorobrachycoma splendida (modificada de Dear, 1979). 43. Sarconesiopsis magellanica (modificada de Dear, 1979). 


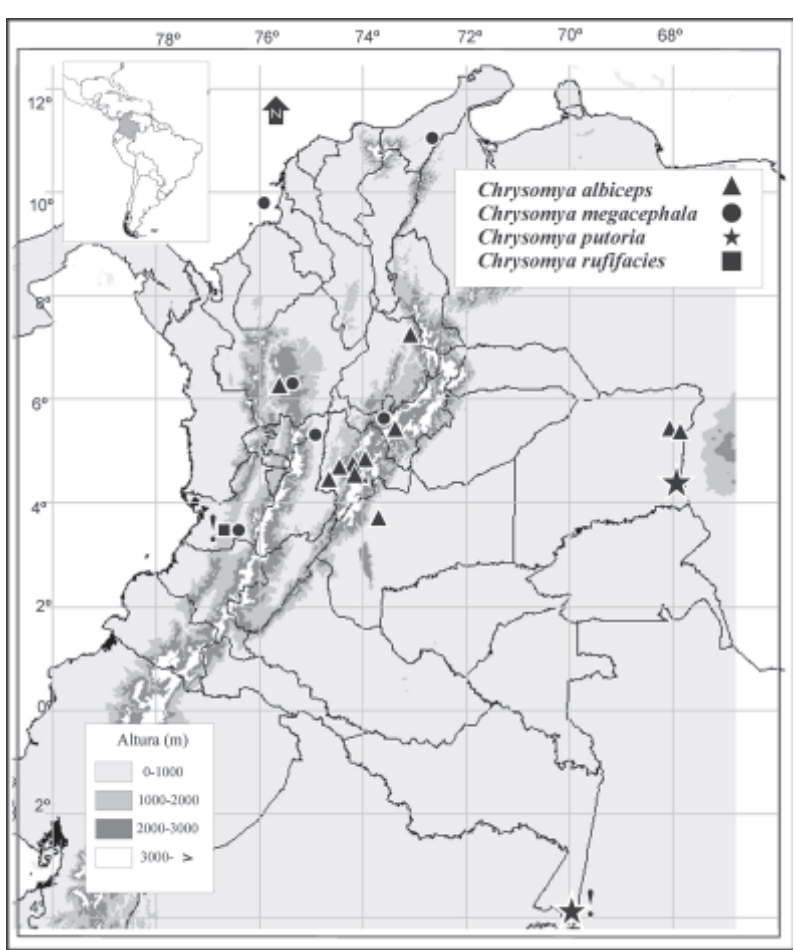

Figura 44. Localidades de las especies del género Chrysomya en Colombia. (!) Registros encontrados en la literatura; Baumgartner y Greenberg (1984) y Barreto et al. (2002).

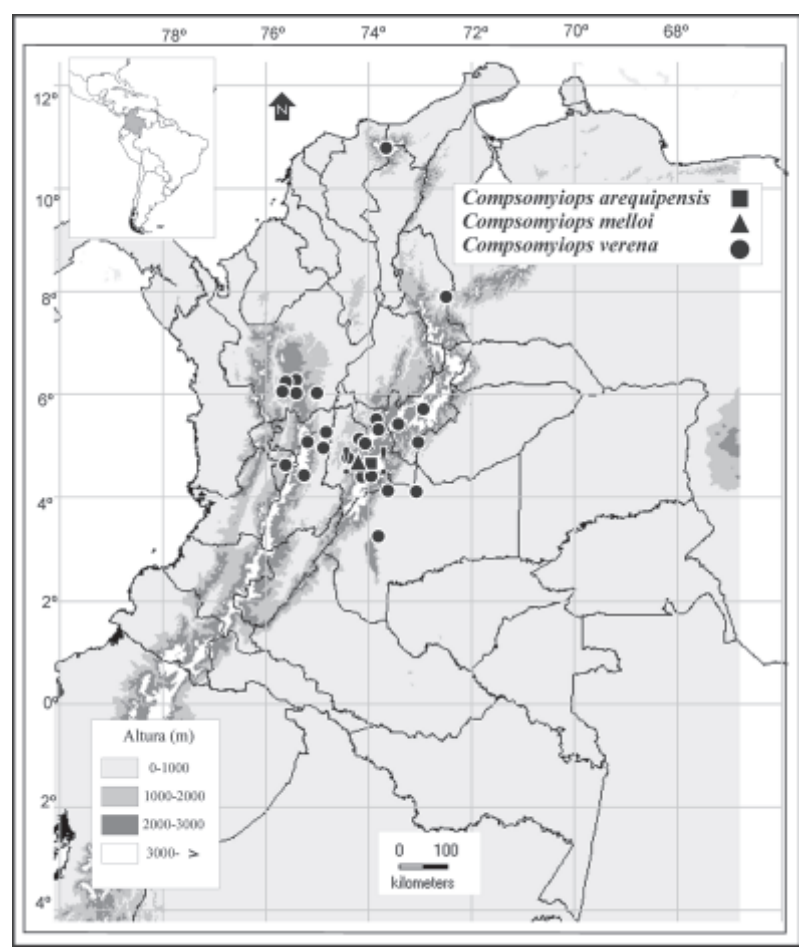

Figura 46. Localidades donde se registran las especies del género Compsomyiops en Colombia. (!) Registros encontrados en la literatura (Dear, 1985).

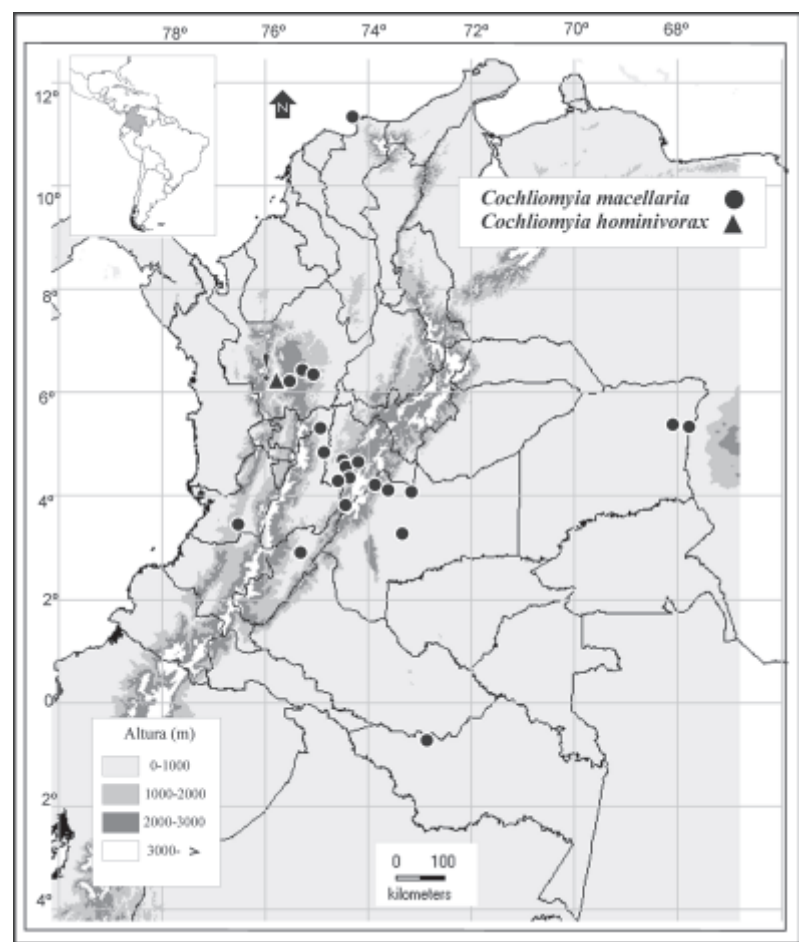

Figura 45. Localidades donde se registra las especies del género Cochliomyia en Colombia. (!) Registro encontrado en la literatura (Pape et al., 2004).

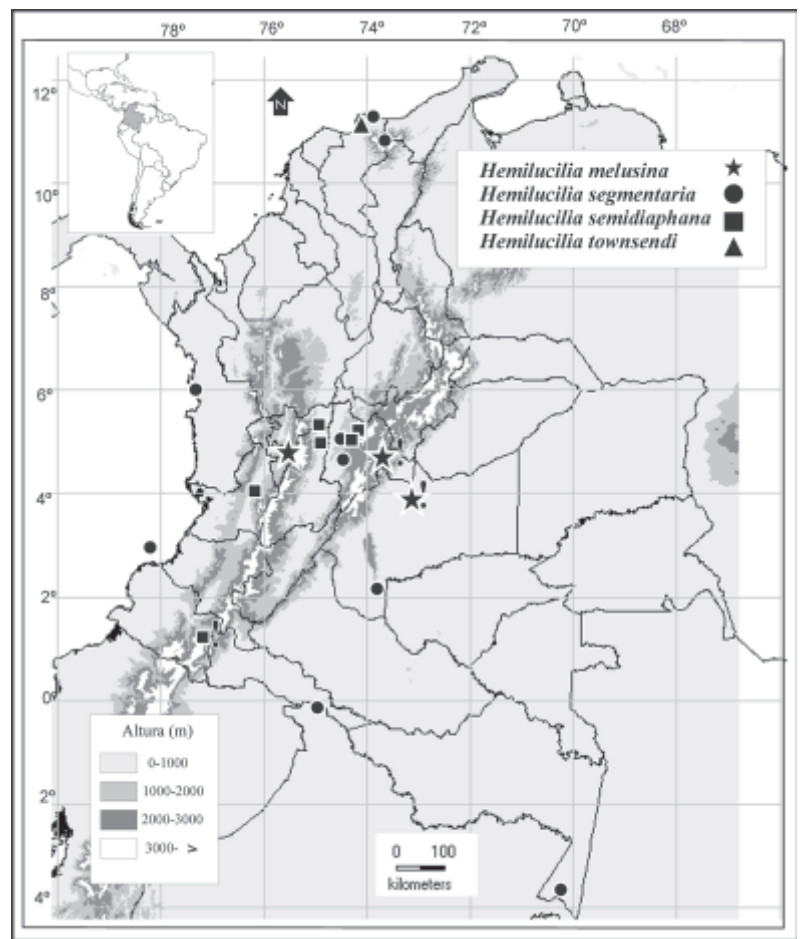

Figura 47. Localidades donde se registran las especies del género Hemilucilia en Colombia. (!) Registros encontrados en la literatura (Dear, 1985). 


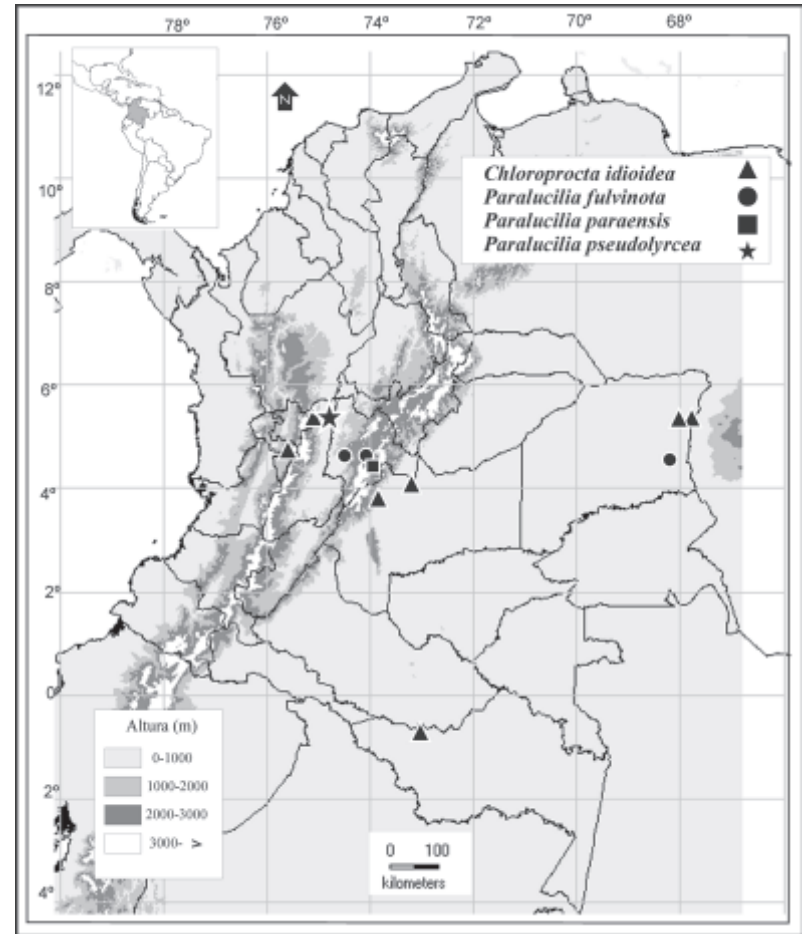

Figura 48. Localidades donde se registran las especies del género Paralucilia y Chloroprocta en Colombia.

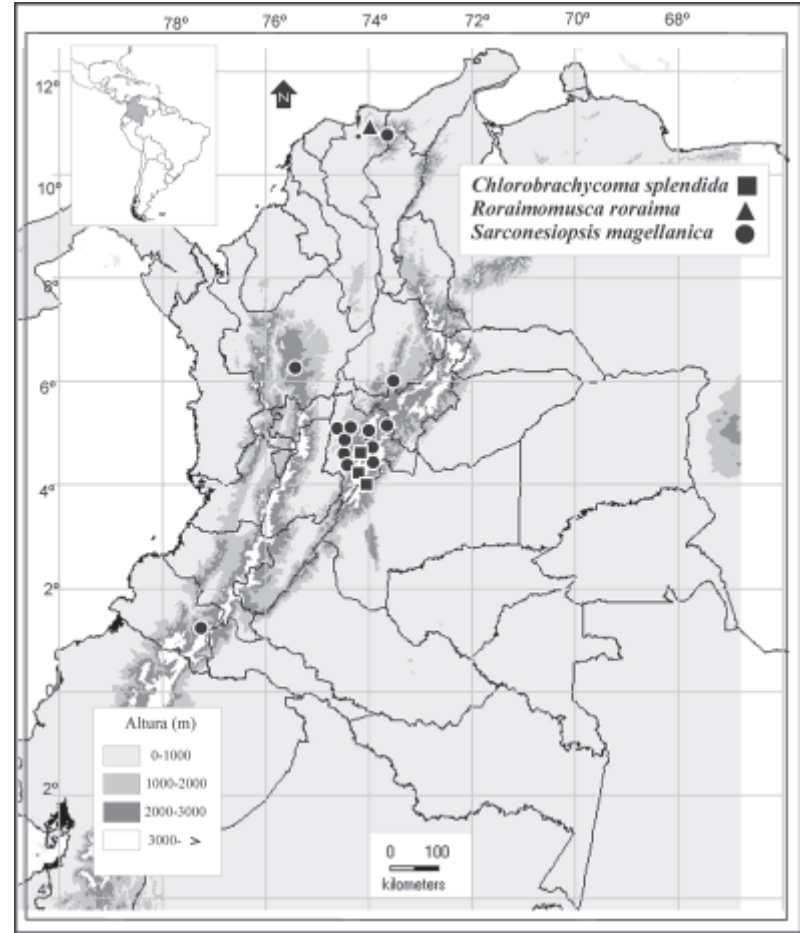

Figura 49. Localidades donde se registran las especies de la subfamilia Toxotarsinae en Colombia. (!) Registro dudoso.

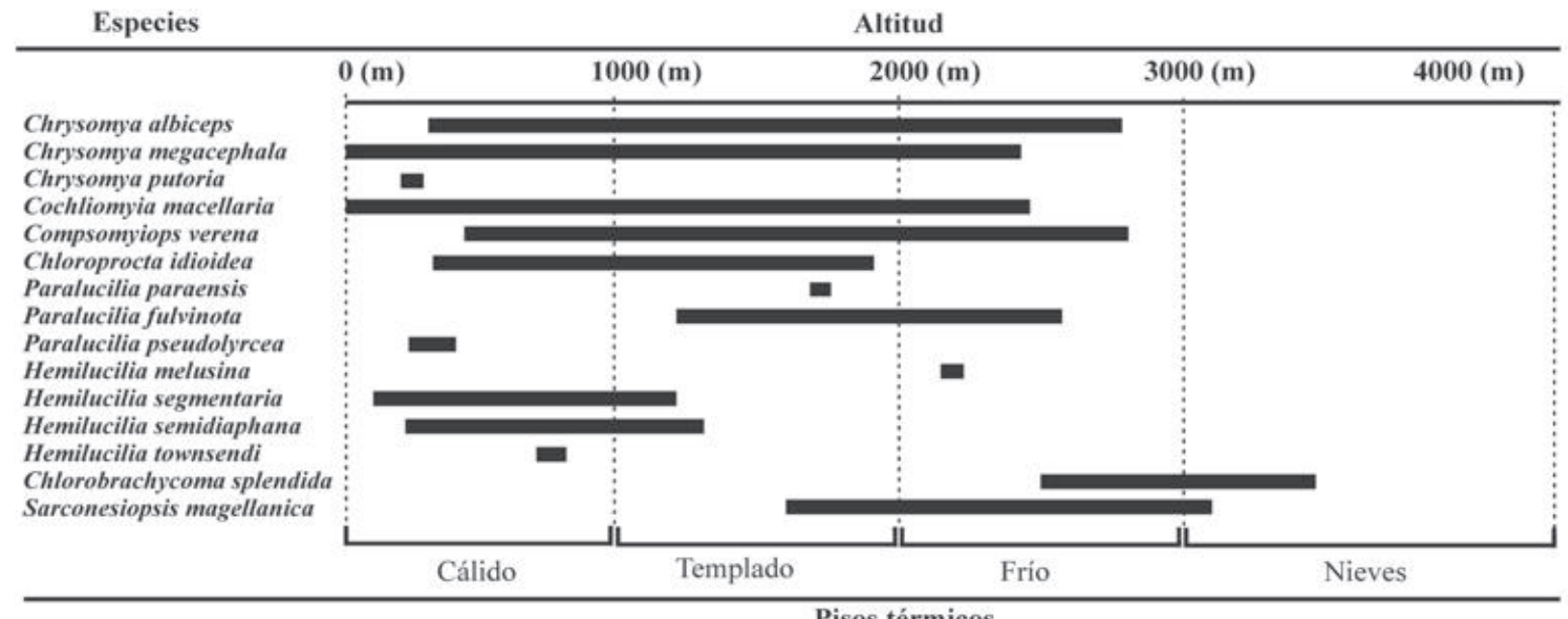

Pisos térmicos

Figura 50. Distribución altitudinal de las especies de califóridos (Chrysominae y Toxotarsinae) registradas en Colombia. 